LETTER

doi:10.1038/nature23478

\title{
Glutamate receptor-like channels are essential for chemotaxis and reproduction in mosses
}

Carlos Ortiz-Ramírez, Erwan Michard, Alexander A. Simon, Daniel S. C. Damineli, Marcela Hernández-Coronado, Jörg D. Becker \& José A. Feijó

This is a PDF file of a peer-reviewed paper that has been accepted for publication. Although unedited, the content has been subjected to preliminary formatting. Nature is providing this early version of the typeset paper as a service to our customers. The text and figures will undergo copyediting and a proof review before the paper is published in its final form. Please note that during the production process errors may be discovered which could affect the content, and all legal disclaimers apply.

Cite this article as: Ortiz-Ramírez, C. et al. Glutamate receptor-like channels are essential for chemotaxis and reproduction in mosses. Nature http://dx.doi.org/10.1038/nature23478 (2017). 


\title{
Glutamate receptor-like channels are essential for chemotaxis and reproduction in mosses
}

\author{
Carlos Ortiz-Ramírez ${ }^{1} \dagger$, Erwan Michard ${ }^{1,2}$, Alexander A. Simon ${ }^{2}$, Daniel S. C. Damineli², Marcela Hernández-Coronado ${ }^{1}$,
} Jörg D. Becker ${ }^{1}$ and José A. Feijó ${ }^{1,2}$

\begin{abstract}
Glutamate receptors are well characterized channels that mediate cell-to-cell communication during neurotransmission in animals. Nevertheless, information regarding their functional role in organisms without nervous systems is still limited. In plants, Glutamate Receptor-like (GLR) genes have been implicated in defence against pathogens, reproduction, control of stomata aperture and light signal transduction ${ }^{1-5}$. However, the numerous GLR genes present in angiosperm genomes $(20 \text { to } 70)^{6}$ has prevented the observation of strong phenotypes in loss-of-function mutants. Here, we show that in the moss Physcomitrella patens, a basal land plant, mutation of GLR genes cause sperm failure in targeting the female reproductive organs. In addition, we show that GLR genes encode non-selective $\mathrm{Ca}^{2+}$ permeable channels that can regulate cytoplasmic $\mathrm{Ca}^{2+}$ and are needed to induce the expression of a BELL1-like transcription factor essential for zygote development. Our work reveals novel functions for GLRs in sperm chemotaxis and transcriptional regulation. Sperm chemotaxis is essential for fertilization in both animals and early land plants like bryophytes and pteridophytes. Therefore, our results are suggestive that ionotropic glutamate receptors may have been conserved throughout plant evolution to mediate cell-to-cell communication during sexual reproduction.
\end{abstract}

Only two GLR genes have been identified in the moss Physcomitrella ${ }^{7}$, and phylogenetic analyses showed that these genes are paralogs to Arabidopsis clade three ${ }^{8}$. As a first approach to elucidate GLR function in mosses, we complemented our previous transcriptomic analysis ${ }^{9}$ to profile the expression of $P p G L R 1$ and PpGLR2. We purified mRNA from all tissues including archegonia, sperm cells, and sporophytes isolated at 3 different developmental stages, and monitored PpGLR1 and PpGLR2 expression by qRT-PCR (Fig.1). PpGLR1 transcript was detected in all tissues, with the highest expression in gametophores and young sporophytes. In contrast PpGLR2 has a very specific expression pattern limited to archegonia, sperm cells, and mature sporophytes (Fig.1a), suggestive of a role in sexual reproduction.

We then proceeded to loss of function studies by generating single and double knock out mutants by homologous recombination (Ppglr1-19, Ppglr2-11; double mutant line referred as Ppglr1/2). Early developmental stages are characterized by the presence of haploid tip growing cells (caulonema and chloronema), and none of our mutant lines displayed any phenotypic defects in these cell types. Even when exposed to low $\mathrm{Ca}^{2+}$ conditions, growth was comparable between wild type ( $\left.w t\right)$ and Ppglr1/2 (Extended Data Fig. 1a). This was unexpected because GLR mutations in Arabidopsis produced tip growth defects in pollen tubes ${ }^{4}$. Since caulonema filaments are the most active tip growing cells in Physcomitrella, we preferentially induced their development to test if tip growth was impaired in Ppglr1/2. However, caulonema cells grew normally (Extended Data Fig. 1b, c), revealing that GLR function in apical growth is not conserved between angiosperms and bryophytes.
Importantly, in Arabidopsis glr mutants pollen tube growth defects lead to male reproductive phenotypes ${ }^{4}$. Having this in mind and considering the transcriptomic profile of PpGLR2, we decided to further investigate if $P p G L R s$ had a conserved role in reproduction. We induced the development of reproductive structures (gametangia) and quantified the production of sporophytes (the diploid zygotic structure) as a way to indirectly assess fertilization rates. Under our conditions, $56.3 \% \pm 11$ of $w t$ gametophores develop mature sporophytes after 6 weeks of induction (Fig. 1c). Remarkably, all Ppglr mutant lines produced far fewer sporophytes compared to $w t$ (Fig. 1b). The percentage of gametophores with sporophytes in Ppglr1 decreased to $33 \% \pm 7$ and was even more severe in Ppglr2 and Ppglr1/2 double knockout, with $17.9 \% \pm 3.9$ and $10.2 \% \pm 6.2$ respectively. Furthermore, the few sporophytes produced by the mutant lines showed retarded maturation (Fig. 1c), suggestive of a possible role in sporophyte developmental progression. Notably, these phenotypes cannot be attributed to defects in the formation of reproductive organs, since archegonia (organs that form the female gametes) and antheridia (the male counterpart) isolated from Ppglr1/2 show the same morphology and developmental patterns as $w t$ (Fig. 1b).

To determine the contribution of male and female gametes to the low sporophyte production rates observed in mutant lines, we carried out cross-fertilization experiments. We used transgenic lines expressing GFP and RFP as markers to discriminate between self-fertilized and hybrid sporophytes. First, we investigated whether sporophyte production rates could increase by crossing GFP-expressing $w t$ plants with Ppglr1/2 mutants. Interestingly, 33\% \pm 6.3 of Ppglr1/2 gametophores produced sporophytes, almost all of them originating from outcrossing events (hybrid sporophytes) (Fig. 1d; Table 1). This observation supports the interpretation that $w t$ sperm can fertilize mutant plants.

Importantly, in this experimental setting complementation depends solely on outcrossing efficiency. Therefore, as a reference we estimated natural outcrossing rates in $w t$ plants and found they were indeed similar to those reported in our first cross (Table 1). To show that Ppglr1/2 archegonia is capable of producing sporophytes at normal rates, we crossed this line with the more efficient outcrosser strain Physcomitrella Villersexel ${ }^{10}$ expressing red fluorescent protein (RFP) (referred to as Vsx RFP from here on). As a result, sporophyte

Table 1 | Sporophyte production rates in cross-fertilization experiments

\begin{tabular}{lccc}
\hline \multirow{2}{*}{ Crossed genotypes } & \multicolumn{3}{c}{$\%$ of gametophores with sporophytes in female line } \\
\cline { 2 - 4 } & From out-cross & From self-fertilization & Total \\
\hline wt GFP of $\times$ wt o & $28.1 \pm 2.8$ & $35.2 \pm 11.6$ & $63.3 \pm 8.8$ \\
wt GFP o $\times$ Ppglr1/2 o & $33 \pm 6.3$ & $0.9 \pm 1.2$ & $33.9 \pm 7.6$ \\
Ppglr1/2 GFP o $\times$ wt o & $1 \pm 1.4$ & $50.2 \pm 6$ & $51.3 \pm 4.6$ \\
\hline
\end{tabular}

50 gametophores were analysed for each cross. Data are mean \pm s.d. from two independent biological experiments. Male and female symbols are used to show how lines were crossed. 
production rates in Ppglr1/2 were even higher than $w t(72 \% \pm 18.9)$ presenting a complete phenotypic rescue (Extended Data Fig. $1 \mathrm{~d}, \mathrm{e}$ ).

In addition, we performed the reverse crossing experiment to prove that Ppglr $1 / 2$ sperm were incapable of achieving fertilization. We found that outcrossing rates dropped drastically when Ppglr 1/2 expressing GFP was crossed as the paternal line, with only $1 \% \pm 1.4$ of $w t$ gametophores producing hybrid sporophytes (Table 1). These results unequivocally demonstrate that the phenotypic transmission of the GLR defect runs through the male by affecting sperm function in Ppglr1/2.

We thus hypothesized that mutant sperm could be defective in the ability to navigate and perceive female cues. An in vitro sperm navigation assay was developed to test this possibility (see materials and methods and Extended Data Fig. 2A). We observed that $w t$ sperm moves with a helicoidal motion at an average speed of $16.7 \pm 3.4 \mu \mathrm{m} / \mathrm{sec}$ (Supplementary Video 1; Fig. 2F). Importantly, a total of $2.6 \pm 1.3$ cells per released cluster (sperm cells are released in single clusters of ca. 150 cells) successfully contacted the archegonia opening, and $1.3 \pm 1.1$ managed to enter the archegonia channel (Supplementary video 3, 4 and Fig. 2a, b, d and e). In contrast, Ppglr1/2 sperm cells move faster than $w t$ at an average speed of $23.2 \pm 10.8 \mu \mathrm{m} / \mathrm{sec}$ (Supplementary Video 2; Fig. $2 \mathrm{~F}$ ) and are far less efficient in targeting and entering the archegonia opening ( $0.63 \pm 1.0$ and $0.54 \pm 0.9$ sperm cells per cluster respectively) (Supplementary Video 5, 6 and Fig. 2. a, c, d and e). Increased speed of mutant sperm cells might reflect their inability to detect and/or respond to female cues. Such responses imply changes in the cell's trajectories that are expected to decrease their linear velocity. Given the random direction of sperm swimming, targeting archegonia is possible just by chance, and, accordingly, none of the successful events from Ppglr1/2 showed sharp changes of direction like it was observed for $w t$. These results implicate $P p$ GLRs in the control of sperm speed and turning motion in response to female cues, suggesting that even small modifications in one of these parameters is sufficient to dramatically reduce fertilization rates.

Because sperm motility and capacitation in animals is controlled in part by rises in free cytosolic $\mathrm{Ca}^{2+}$ concentration $\left(\left[\mathrm{Ca}^{2+}\right] \text { cyt }\right)^{11}$, we decided to investigate if $P p$ GLRs could regulate $\left[\mathrm{Ca}^{2+}\right]$ cyt in moss sperm. We loaded $w t$ and mutant sperm with the cytoplasmic $\mathrm{Ca}^{2+}$ indicator fluo-4-AM and expose the cells to medium containing numerous archegonia. Cytoplasmic $\left[\mathrm{Ca}^{2+}\right]$ was lower in sperm cells lacking GLRs $(p<0.001)$, indicating these channels may affect sperm cell function through the regulation of $\mathrm{Ca}^{2+}$ concentration (Fig. 2G). To investigate this function further, we co-expressed $P p G L R 1$ (using the expression vector pCI-PpGLR1) with the $\mathrm{Ca}^{2+}$ genetic probe Yellow CaMeleon 3.6 (YC3.6) in mammalian COS-7 cells. Cells transfected with pEF1-YC3.6 plus pCI-Pp GLR1 had higher $\left[\mathrm{Ca}^{2+}\right]$ cyt than control cells transfected with pEF1-YC3.6 plus pCI empty vector (Fig. $2 \mathrm{H}$, statistics in Extended data Fig. 2B). Moreover, a clear increase in $\left[\mathrm{Ca}^{2+}\right]$ cyt was observed in PpGLRlexpressing cells when $14.5 \mathrm{mM} \mathrm{Ca}^{2+}$ was added to the bath solution (originally containing $1 \mathrm{mM}$ EGTA) (Fig. 2H, I). These responses were recorded in the absence of any potential ligand in the bath solution, demonstrating a basal activity of PpGLR1. The addition of the cation channel blocker gadolinium $\left(\mathrm{Gd}^{3+}\right)$ at $1 \mathrm{mM}$ abolished the response to external $\mathrm{Ca}^{2+}$ (Fig. $\left.2 \mathrm{H}, \mathrm{I}\right)$. Note that $\mathrm{Gd}^{3+}$ did not decrease basal $\left[\mathrm{Ca}^{2+}\right] \mathrm{cyt}$, which remained higher than in control cells (Fig. $2 \mathrm{H}, \mathrm{I}$ ), suggesting that the kinetics of cytosolic $\mathrm{Ca}^{2+}$ extrusion and basal $\left[\mathrm{Ca}^{2+}\right]$ cyt adjustment in COS-7 cells are significantly slower than our 7-minute-long experiment.

While the previous results are sufficient to explain the reduced rates of fertilization observed on Ppglr1/2, defects in sporophyte maturation remained unaccounted for. As part of their normal maturation process, sporophytes undergo meiosis to produce large numbers of spores. To determine if spore production was affected in the loss-of-function mutant, we isolated and analysed their size, morphology and numbers. Ppglr1/2 sporophytes produced smaller spores and in fewer numbers (Fig.3 a,b,c), indicating a role in sporophyte development. Interestingly, there is evidence that some ion channels regulating $\mathrm{Ca}^{2+}$ signalling events can affect development by inducing the expression of transcription factors ${ }^{12,13}$. Using microarrays, we analysed the transcriptome of wt and Ppglr1/2 reproductive organs isolated 16 days after gametangia induction. At this time, early embryos are expected to develop ${ }^{9}$. Microarray analysis revealed that several transcripts were significantly up and down-regulated in Ppglr1/2, implicating GLR activity in the modulation of gene expression. Although it was not possible to distinguish in which reproductive structure the transcripts were produced, we found the sporophyte-associated BELL1 transcription factor homolog among the most significantly down regulated genes (Extended Data Table 1, and Supp Table 2). Members of the BELL1 family are known to control egg cell and sporophyte development in Arabidopsis ${ }^{14-17}$, as well as the regulation of the diploid genetic program in Chlamydomonas and Physcomitrella ${ }^{18,19}$. In light of these results, we hypothesized that the low levels of PpBELL1 detected in the mutant were responsible for the sporophyte developmental defects. To test this hypothesis, we attempted to rescue Ppglr1/2 by driving the expression of $P p$ BELL1 with the promoter of $P p$ GLR2. This promoter is specifically expressed in reproductive organs and sporophytes, preventing possible artefacts of over-expression at other developmental stages. Using homologous recombination, the PpBELL1 coding sequence was inserted in situ immediately downstream of the PpGLR2 promoter. This line is referred to as $P p G L R 2:: P p B E L L 1$. In accordance with our hypothesis, sporophyte analyses of $P$ PGLR2::P PBELL1 showed a significant increase in the number of spores per sporophyte as well as in spore area, which increased from $200.9 \mu \mathrm{m}^{2} \pm 66$ in Ppglr1/2 to $428.1 \mu \mathrm{m}^{2} \pm$ 185 in PpGLR2::PpBELL1 (Fig. 3b, c). This rescue indicates not only that $P p B E L L 1$ gene plays an important role in sporophyte development, but also that $P p G L R 2$ may act upstream of a signalling pathway that regulates the expression of this transcription factor. In line with this, gene network analysis performed using the PlaNET tool ${ }^{20}$ showed that many transcription factor genes are associated with $P p G L R 2$, but not with PpGLR1 (Extended Data Fig. 3). Most of them have homologs known to control sporophyte development in angiosperms. Gene ontology analysis confirmed that the PpGLR2 gene network is mainly associated with transcriptional regulation and protein kinase activity (Extended Data Fig. 3 \& Supplementary Table 3). Importantly, sporophyte production rates did not increase in PpGLR2::PpBELL1 complementation line (Extended Data Fig. 2C) showing that $P p G L R$ activity affects fertilization and sporophyte development through independent mechanisms.

Finally, given their conspicuous phenotypic effects in reproduction, we further aimed at establishing the function of Physcomitrella GLRs as ionotropic channels using the patch-clamp technique. We first investigated if $P p G L R 1$ was present on the plasma membrane of moss cells by measuring whole-cell ion currents in protonema isolated protoplasts (Fig. 4). Under our conditions (see materials and methods) instantaneous currents were significantly higher in $w t$ protoplast when compared to Ppglr1/2 from -100 to $+60 \mathrm{mV}$ (Fig.4a,b). Moreover, protoplasts stably overexpressing PpGLR1 cDNA under the control of the 35S promoter exhibited significantly stronger currents than $w t$, (Fig.4a,b). These currents were inhibited by $\mathrm{Gd}^{3+}$ (0.5-1.0 mM; Extended Data Fig. $4 \mathrm{a}, \mathrm{b})$ and by the iGluR antagonists CNQX and AP5 $(50 \mu \mathrm{M}$ and $200 \mu \mathrm{M}$ respectively) (Fig. $4 \mathrm{c}$ and $\mathrm{d}$ ).

We further characterized $P p$ GLR1 conductivity in mammalian COS-7 cells. Cells expressing PpGLR1 had higher currents than control cells (approximately $300 \mathrm{pA}$ at $-100 \mathrm{mV}$ ) (Fig. 4e and f) and they were inhibited by the same iGluR antagonists used in protoplasts (Extended Data Fig $4 \mathrm{c}$ and d; and Extended Data Fig. 5 e, f). Cationic conductance increased in both COS-7 cells and protoplasts upon extracellular $\mathrm{Na}^{+}$elevation (10 to $100 \mathrm{mM}$ ), suggesting weak cationic selectivity (Extended Data Fig. 6). However, when calcium and chloride were used as the only charge carriers in the solution and perfused in increasing concentrations $(3,10,30$ and $70 \mathrm{mM})$, a clear current activation was observed demonstrating $\mathrm{Ca}^{2+}$ permeability of PpGLR1 (Fig. $4 \mathrm{~g}, \mathrm{~h}$ ). 
We thus propose that both sperm chemotaxis and transcriptional modulation of $P$ BBELL1 are dependent on GLR-mediated regulation of $\left[\mathrm{Ca}^{2+}\right]$ cyt. Since expression of several ionotropic GLRs has been detected in sperm cells of plants belonging to different phylogenetic groups $^{21}$, as well as in mammalian sperm ${ }^{22}$, glutamate receptors may have a conserved role in male gamete signal perception/steering common to bryophytes, vascular plants and animals ${ }^{4}$.

Online Content Methods, along with any additional Extended Data display items and Source Data, are available in the online version of the paper; references unique to these sections appear only in the online paper.

Received 28 December 2016; accepted 14 July 2017.

Published online 24 July 2017.

1. Forde, B. G. \& Roberts, M. R. Glutamate receptor-like channels in plants: a role as amino acid sensors in plant defence? F1000prime reports 6,37 , doi:10.12703/P6-37 (2014).

2. Cho, D. et al. De-regulated expression of the plant glutamate receptor homolog AtGLR3.1 impairs long-term Ca2+-programmed stomatal closure. The Plant journal : for cell and molecular biology 58, 437-449, doi:10.1111/j.1365-313X.2009.03789.x (2009)

3. Lam, H. M. et al. Glutamate-receptor genes in plants. Nature 396, 125-126, doi:10.1038/24066 (1998).

4. Michard, E. et al. Glutamate receptor-like genes form $\mathrm{Ca} 2+$ channels in pollen tubes and are regulated by pistil D-serine. Science 332, 434-437, doi:10.1126/science.1201101 (2011)

5. Mousavi, S. A Chauvin, A. Pascaud, F. Kellenberger, S. \& Farmer, E. E. GLUTAMATE RECEPTOR-LIKE genes mediate leaf-to-leaf wound signalling. Nature 500, 422-426, doi:10.1038/nature12478 (2013).

6. Chiu, J. C. et al. Phylogenetic and expression analysis of the glutamatereceptor-like gene family in Arabidopsis thaliana. Molecular biology and evolution 19, 1066-1082 (2002).

7. Verret, F., Wheeler, G., Taylor, A. R., Farnham, G. \& Brownlee, C. Calcium channels in photosynthetic eukaryotes: implications for evolution of calcium-based signalling. The New phytologist 187, 23-43, doi:10.1111/j.1469-8137.2010.03271.x (2010)

8. De Bortoli, S., Teardo, E., Szabo, I., Morosinotto, T. \& Alboresi, A. Evolutionary insight into the ionotropic glutamate receptor superfamily of photosynthetic organisms. Biophysical chemistry 218, 14-26, doi:10.1016/j.bpc.2016.07.004 (2016).

9. Ortiz-Ramirez, C. et al. A Transcriptome Atlas of Physcomitrella patens Provides Insights into the Evolution and Development of Land Plants. Molecular plant 9, 205-220, doi:10.1016/j.molp.2015.12.002 (2016).

10. Perroud, P. F., Cove, D. J., Quatrano, R. S. \& McDaniel, S. F. An experimental method to facilitate the identification of hybrid sporophytes in the moss Physcomitrella patens using fluorescent tagged lines. The New phytologist 191 , 301-306, doi:10.1111/j.1469-8137.2011.03668.x (2011).

11. Ren, D. J. et al. A sperm ion channel required for sperm motility and male fertility. Nature 413, 603-609, doi:Doi 10.1038/35098027 (2001)

12. Lilienbaum, A. \& Israel, A. From Calcium to NF- B Signaling Pathways in Neurons. Molecular and Cellular Biology 23, 2680-2698, doi:10.1128/ mcb.23.8.2680-2698.2003 (2003).

13. Hogan, P. G., Chen, L., Nardone, J. \& Rao, A. Transcriptional regulation by calcium, calcineurin, and NFAT. Genes \& development 17, 2205-2232, doi:10.1101/gad.1102703 (2003).

14. Rutjens, B. et al. Shoot apical meristem function in Arabidopsis requires the combined activities of three BEL1-like homeodomain proteins. The Plant journal : for cell and molecular biology 58, 641-654, doi:10.1111/j.1365-313X.2009.03809.x (2009).

15. Reiser, L. et al. The BELL1 gene encodes a homeodomain protein involved in pattern formation in the Arabidopsis ovule primordium. Cell 83, 735-742 (1995)

16. Brambilla, V. et al. Genetic and molecular interactions between BELL1 and MADS box factors support ovule development in Arabidopsis. The Plant cell 19 2544-2556, doi:10.1105/tpc.107.051797 (2007)
17. Bowman, J. L., Sakakibara, K., Furumizu, C. \& Dierschke, T. Evolution in the Cycles of Life. Annual review of genetics 50, 133-154, doi:10.1146/annurevgenet-120215-035227 (2016).

18. Horst, N. A. et al. A single homeobox gene triggers phase transition, embryogenesis and asexual reproduction. Nature Plants 2, 15209 , doi:10.1038/nplants.2015.209 (2016).

19. Lee, J. H., Lin, H., Joo, S. \& Goodenough, U. Early sexual origins of homeoprotein heterodimerization and evolution of the plant KNOX/BELL family. Cell 133, 829-840, doi:10.1016/j.cell.2008.04.028 (2008).

20. Mutwil, M. et al. PlaNet: combined sequence and expression comparisons across plant networks derived from seven species. The Plant cell 23,895-910, doi:10.1105/tpc.111.083667 (2011).

21. Borges, F. et al. Comparative transcriptomics of Arabidopsis sperm cells Plant physiology 148, 1168-1181, doi:10.1104/pp.108.125229 (2008).

22. Hu, J. H. et al. Identification of glutamate receptors and transporters in mouse and human sperm. Journal of andrology 25, 140-146 (2004).

23. Ashton, N. \& Cove, D. The Isolation and Preliminary Characterisation of Auxotrophic and Analogue Resistant Mutants of the Moss, Physcomitreila patens. Molecular Genetics and Genomics, 9 (1977).

24. Reski, R. \& Abel, W. Induction of budding on chloronemata and caulonemata of the moss, Physcomitrella patens, using isopentenyladenine. Planta, $\mathbf{5}$ (1985).

25. Hohe, A., Rensing, S. A., Mildner, M., Lang, D. \& Reski, R. Day Length and Temperature Strongly Influence Sexual Reproduction and Expression of a Novel MADS-Box Gene in the Moss Physcomitrella patens. Plant Biology, $\mathbf{8}$ (2002).

26. Reyes, R. et al. Cloning and Expression of a Novel pH-sensitive Two Pore Domain $\mathrm{K}+$ Channel from Human Kidney. Journal of Biological Chemistry 273. 30863-30869, doi:10.1074/jbc.273.47.30863 (1998).

27. Jurman, M. E., Boland, L. M., Liu, Y. \& Yellen, G. Visual identification of individual transfected cells for electrophysiology using antibody-coated beads. BioTechniques, 6 (1994)

28. Li, C. \& Wong, W. H. Model-based analysis of oligonucleotide arrays: expression index computation and outlier detection. Proc Natl Acad Sci U S A 98, 31-36, doi:10.1073/pnas.011404098 (2001).

29. Huang da, W., Sherman, B. T. \& Lempicki, R. A. Systematic and integrative analysis of large gene lists using DAVID bioinformatics resources. Nature protocols 4, 44-57, doi:10.1038/nprot.2008.211 (2009).

Supplementary Information is available in the online version of the paper.

Acknowledgements Thanks to P. F. Perroud (Phillips University, Marburg) for the Gransden GFP and Villersexe/ RFP lines; L. Dolan and T. Tan (Oxford University) for pBNRF, $\mathrm{pBH}$ rev and $\mathrm{p} 108 \mathrm{GW} 35$ s vectors; M. Hasebe (NIBB, Okazaki) for pTN83 construct; S. Rensing (University of Marburg) for the Nimblegen_Ppat_SR_-exp_HX12 array and the IRB/ FGC (Barcelona) for microarray processing; J. Kudla (University of Muenster) for the pEF1-YC3.6 vector. C.O.R. and M.H.C. acknowledge fellowships from MarieCurie ITN-Plant Origins (FP7-PEOPLE-ITN-2008). J.A.F. acknowledges grants from FCT-Portugal (BEX-BCM/0376/2012; PTDC/BIA-PLA/4018/2012) and NSF-US (MCB 1616437/2016).

Author Contributions C.O.R. was involved in generating all experimental data except electrophysiology. E.M. and A.A.S. performed the electrophysiology. D.S.C.D. performed image and data analysis. M.H.C. and J.D.B. isolated sperm cells, purified RNA and analysed transcriptomic data. J.A.F. conceived the project. J.A.F. and C.O.R. wrote the article and conceived all experiments. All authors discussed and improved the manuscript.

Author Information Reprints and permissions information is available at www.nature.com/reprints. The authors declare no competing financial interests. Readers are welcome to comment on the online version of the paper. Publisher's note: Springer Nature remains neutral with regard to jurisdictiona claims in published maps and institutional affiliations. Correspondence and requests for materials should be addressed to J.A.F. (jfeijo@umd.edu).

Reviewer Information Nature thanks J. Kudla and the other anonymous reviewer(s) for their contribution to the peer review of this work. 


\section{RESEARCH LETTER}

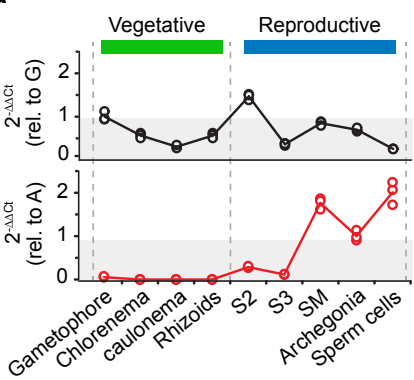

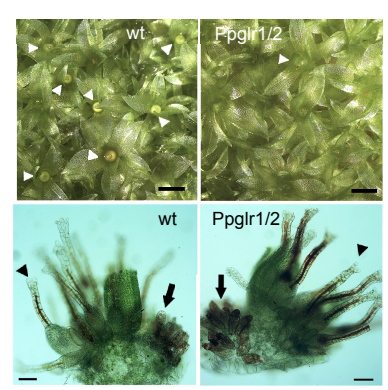

d

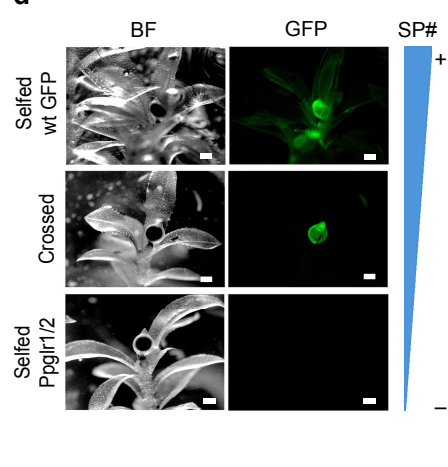

Figure 1 | PpGLR mutants have male reproductive phenotypes. (a) Expression levels of PpGLR1 (red line) and PPGLR2 (black line) were determined by qRT-PCRs. Fold change expression values relative to gametophore and archegonia are shown. $\mathrm{S} 2=9$ day old sporophyte (d.o.s), $\mathrm{S} 3=18$ d.o.s., $\mathrm{SM}=28$ d.o.s. RNA from 3 biological replicates was used. Bold lines display the mean values from three technical replicates (open circles). (b) $w t$ gametophores show more sporophytes than Ppglr1/2 (arrowheads) (upper panels). Images of archegonia (arrowheads) and antheridia (arrows) (lower panel) show that reproductive organs have normal development. (scale bars, $1 \mathrm{~mm}$ upper and $50 \mu \mathrm{m}$ lower panel; representative of 3 images). (c) Quantification of sporophyte production in different genotypes. 100 gametophores were counted per sample. Data from several independent biological replicates. $n=12(w t), n=8$ (Ppglr1), $n=7$ (Ppglr2), $n=8$ (Ppglr1/2). $P$ values shown were obtained from unpaired two-tailed $t$-tests. Box plots show 25, 75 percentile and lines indicate outliers. (d) Self-fertilization events (upper and lower panels) and outcrossing events (middle panel) were identified in cross-fertilization experiments between $w t$ GFP (paternal line) and Ppglr1/2 (maternal line). Sporophyte number (SP\#) decreased drastically in selfed Ppglr1/2 (Scale bars correspond to $300 \mu \mathrm{m}$; representative of 4 images). 
a
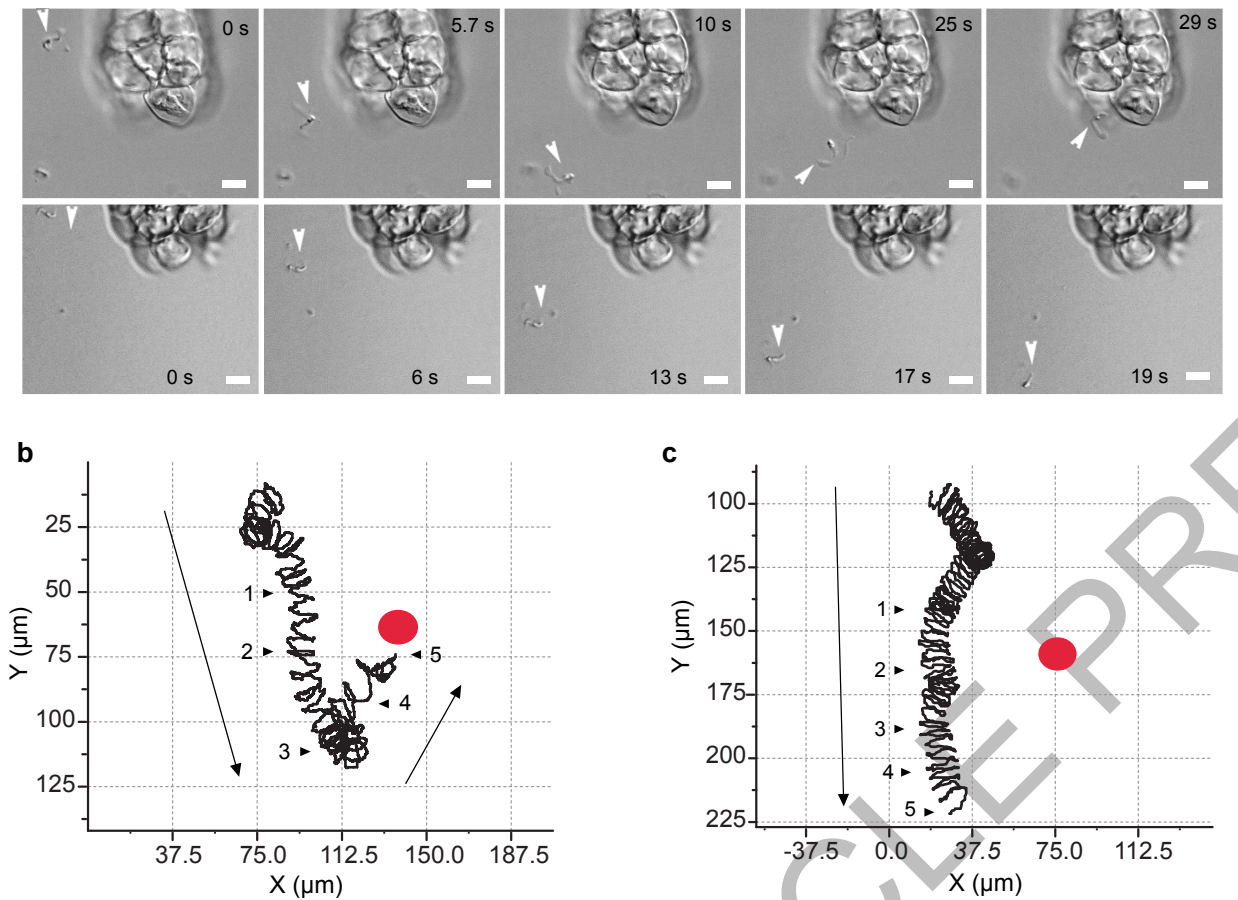

C

d

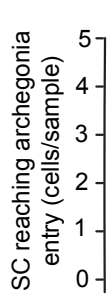

e
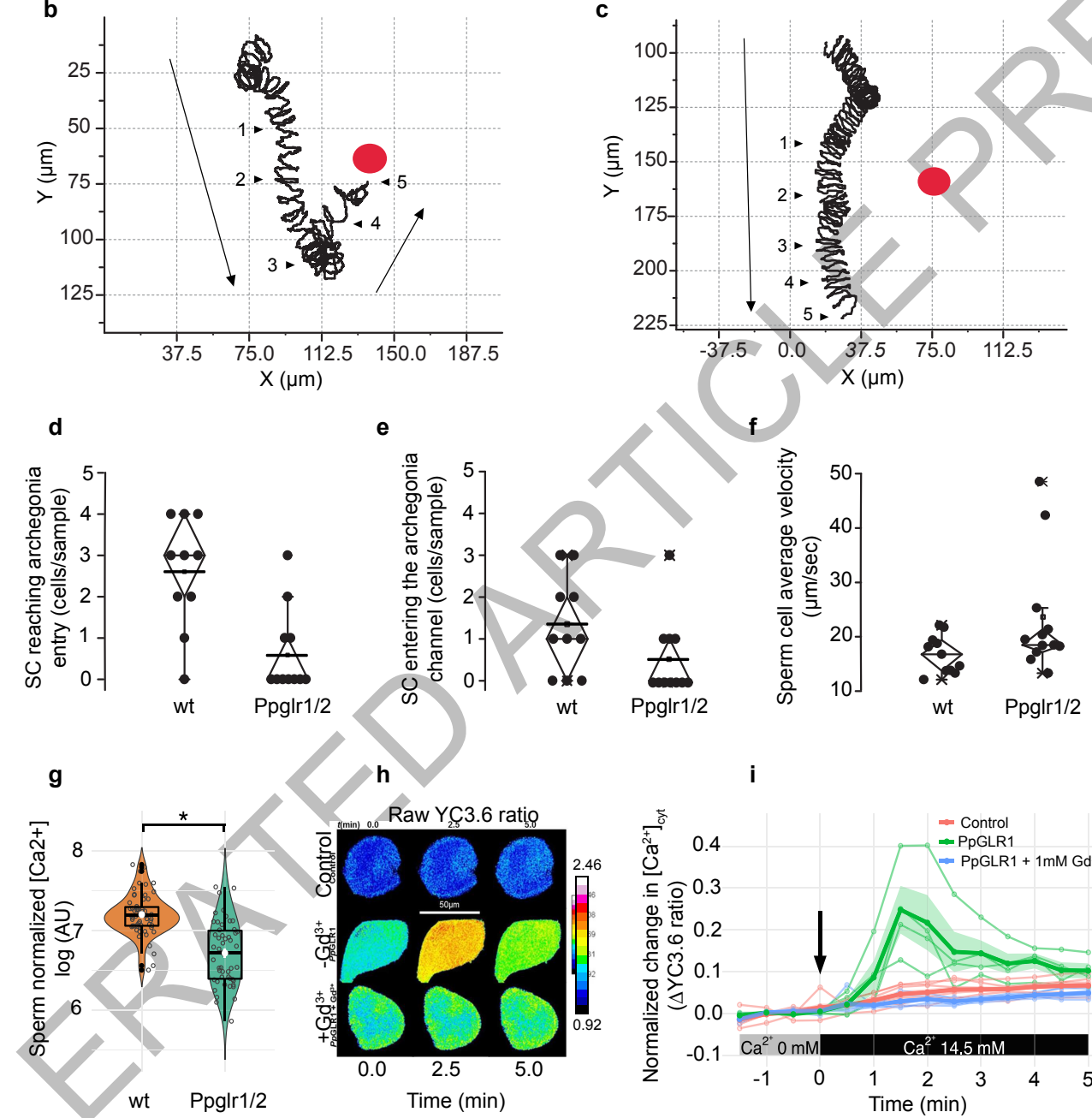

f

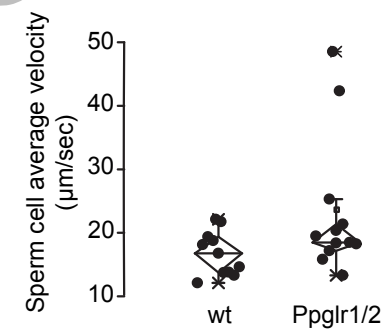

i

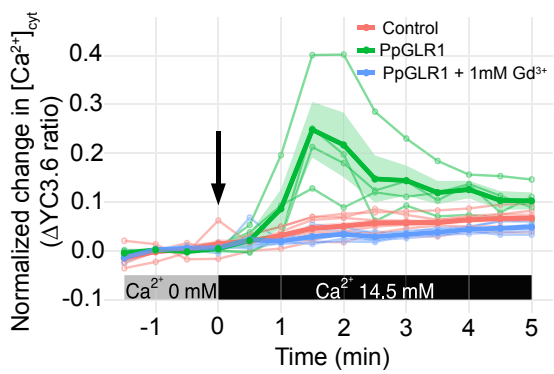

Figure 2 | Sperm cells from Ppglr1/2 fail to reach the archegonia entrance. (a) Sperm cell swimming behaviour in $w t$ (upper panel) and Ppglr1/2 (lower panel). Arrowheads indicate sperm position and direction (scale bar corresponds to $10 \mu \mathrm{m}$; representative frames of 5 similar videos). (b-c) Comparison between $w t$ (b) and Ppglr1/2 (c) sperm cell trajectories. (representative of 10 independent biological replicates). Numbers indicate sperm cell position when frames presented in (a) were recorded, corresponding to frames 1 to 5 from left to right. Red circles represent archegonia entrance and arrows indicate sperm forward movement direction. (d) Number of sperm cells reaching the archegonia entrance. wt sperm was more efficient at reaching archegonia ( $p=0.001$, two-tailed unpaired $t$-test). $n=10(w t), n=11$ (Ppglr1/2). (e) $w t$ sperm cells are also more successful at entering into the archegonia opening ( $p=0.05$, MannWhitney rank sum test). $n=10(w t), n=11$ (Ppglr1/2). (f) Mutant sperm cells move faster than $w t$ sperm ( $p=0.037$, one-tailed unpaired $t$-test). $n=11(w t), n=12($ Ppglr1/2). Biological replicates. Bold lines in $\mathbf{d}, \mathbf{e}$ and f represent mean values. (g) Cytoplasmic $\mathrm{Ca}^{2+}$ concentration is higher in $w t \operatorname{sperm}\left(p=6 \times 10^{-11}\right.$, unequal variances $t$-test). The peak of the signal

for each cell is shown in grey circles for $w t . n=59(w t), n=61$ (Ppglr1/2), technical replicates. Violin plots show curves of density estimates, boxplots and white points with lines as mean and standard errors. (h) Cytosolic $\mathrm{Ca}^{2+}$ concentration monitored with Yellow CaMeleon 3.6 (raw YC3.6 ratio). Typical YC3.6 ratio for control cells (top, representative of 7 images), cells expressing PpGLR1 without (middle, representative of 4 images) or with $\mathrm{Gd}^{3+}$ ( $1 \mathrm{mM}$ ) (bottom, representative of 7 images) at time $0,2.5$, and $5 \mathrm{~min}$ after the addition of $\mathrm{Ca}^{2+}(14.5 \mathrm{mM})$ (colour wedge depicts raw YC3.6 ratio). (I) Time course of the change in cytosolic $\mathrm{Ca}^{2+}$ before and after the addition of $\mathrm{Ca}^{2+}$ in the bath (indicated by the arrow). The $y$-axis shows values of YC3.6 ratio (cp-Venus/CFP) minus the average ratio prior to the $\mathrm{Ca}^{2+}$ increase. Cells expressing PpGLR1 (green line, $n=4$ ), control cells, (blue line, $n=7$ ), and cells expressing PpGLR1 exposed to $\mathrm{Gd}^{3+}(1 \mathrm{mM})$ (red line, $n=7$ ) are shown. The bold lines display the averaged response for each treatment with standard error represented by the shaded area, and thin lines individual replicates. EGTA $(1 \mathrm{mM})$ was added to the medium without $\mathrm{Ca}^{2+}$. 


\section{RESEARCH LETTER}

a

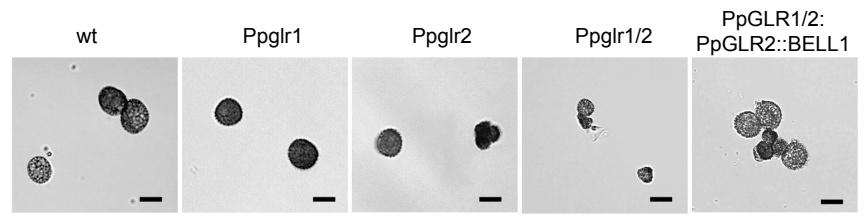

b

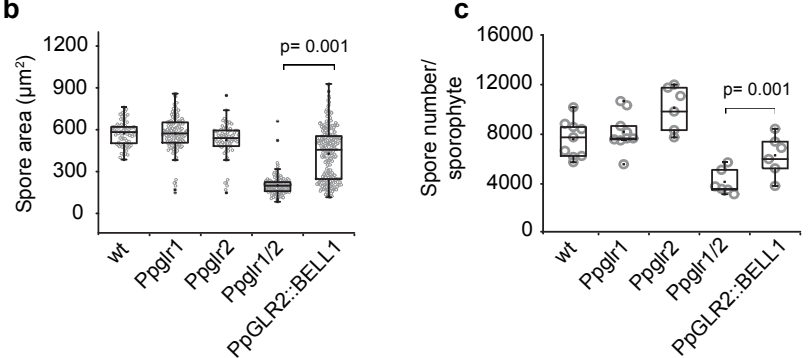

Figure $3 \mid P p B E L L 1$ influence sporophyte spore production and size. (a). Pictures of isolated spores showing that Ppglr1/2 produced smaller spores than $w t$. Some of them presented irregular shapes and form masses of "glued" spores (representative of at least 20 images).

(b) Measurements of spore area. Spores from 3 biological replicates were pooled and measured. Mean \pm s.d. from individually measured spores. $n=82(w t), n=62$ (Ppglr1), $n=75$ (Ppglr2), $n=173$ (Ppglr1/2), and $n=284$ (PpGLR2::BELL1). (c) Quantification of the number of spores per sporophyte showed Ppglr1/2 produced less spores. Mean \pm s.d. from several biological replicates. $n=9(w t), n=6$ (Ppglr1, Ppglr2, Ppglr1/2, $P p$ GLR2::BELL1). Complementation of Ppglr1/2 (last bar in B and C) caused a significant increase in spore size and numbers. Shown $P$ values were obtained from Mann-Whitney $U$ test and $t$-test for spore area and spore numbers respectively. (scale bars correspond to $25 \mu \mathrm{m}$ ). Box plots in b and $\mathbf{c}$ show 25,75 percentile and lines indicate outliers. 
a

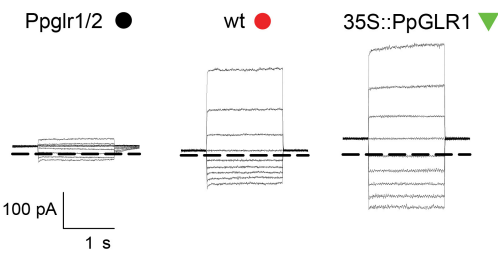

c

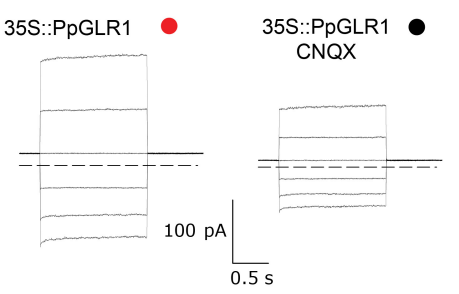

b

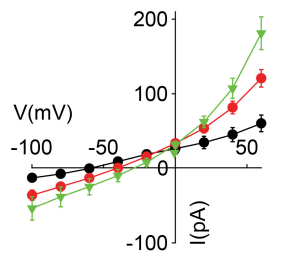

d

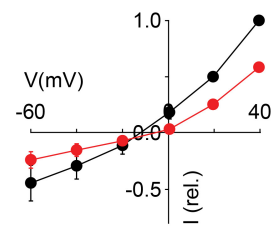

h

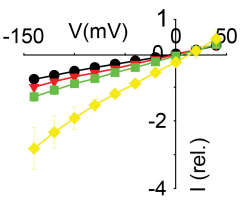

$100 \mathrm{pA} \underset{0.5 \mathrm{~s}}{\longleftarrow}$
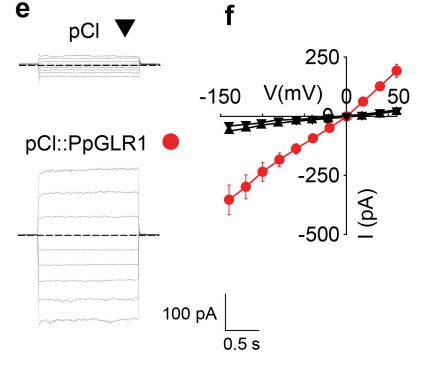

$\mathrm{Ca}^{2+} 3 \mathrm{mM}$

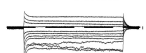

$\mathrm{Ca}^{2+} 30 \mathrm{mM}$

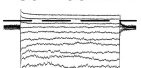

$\mathrm{Ca}^{2+} 70 \mathrm{mM}$

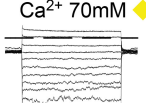

Figure $4 \mid$ PpGLRs are $\mathrm{Ca}^{2+}$-permeable channels in the plasma membrane. (a) Typical currents recorded by patch-clamp (wholecell configuration) in protoplasts from protonema of Ppglr1/2,wt and 35S::Pp GLR1 over-expressor (see methods). (b) Current/voltage curves of stationary currents recorded in experiments shown in a. Black circlesPpglr1/2 $(n=19)$, red circles- $w t(n=40)$, green triangles- 35S::PpGLR1 $(n=20)$. (c) Effect of CNQX $(50 \mu \mathrm{M})$ on typical currents recorded in protoplasts from 35S::PpGLR1 $(n=5)$. (d) Respective standardized current/voltage before (black circles) and after (red circles) application of CNQX, $(n=5)$. (e) Typical currents recorded in COS-7 cells transfected with the pCI empty vector (top) and pCI-PpGLR1 (bottom) recorded in standard COS-7 cell solutions (see methods). (f) Current/ voltage curves of control COS-7 cells transfected with the screening pIRES-CD8 vector (black triangle up, $n=5$ ) or with pIRES-CD8 plus pCI empty vector (black triangle down, $n=7$ ), and cells transfected with pIRES-CD8 plus pCIPpGLR1 (red circles, $n=13$ ). (g) Typical currents recorded from COS-7 cells transfected with pCI-PpGLR1 under successive perfusion of external solutions with increasing $\mathrm{Ca}^{2+}$ concentrations $(3,10,30$ and $70 \mathrm{mM})$ while maintaining constant $\mathrm{Cl}^{-}$. (H) Standardized current/voltage curves of experiments presented in $\mathbf{G}$. $\mathrm{Ca}^{2+} 3 \mathrm{mM}$ (black circles, $\mathrm{n}=5$ ), $\mathrm{Ca}^{2+}$ $10 \mathrm{mM}$ (red triangle, $\mathrm{n}=5$ ), $\mathrm{Ca}^{2+} 30 \mathrm{mM}$ (green square, $\mathrm{n}=5$ ), $\mathrm{Ca}^{2+} 70 \mathrm{mM}$ (yellow diamond, $\mathrm{n}=3$ ). All error bars represent the standard error of the mean. In all cases $n$ represents biological replicates. 


\section{METHODS}

Plant Material and Growth Conditions. The Gransden wt strain from Physcomitrella patens Bruch \& Schimp ${ }^{23}$ was used for this study. Plant material was routinely grown on Petri dishes containing KNOPS media ${ }^{24}$ supplemented with $0,5 \mathrm{~g} / \mathrm{L}$ ammonium tartrate dibasic and $5 \mathrm{~g} / \mathrm{L}$ glucose (Sigma-Aldrich Co, St. Louis, US) at $25^{\circ} \mathrm{C}, 50 \%$ humidity and $16 \mathrm{~h}$ light (light intensity $90-100 \mu \mathrm{mol} \mathrm{m}-2 \mathrm{~s}-1$ ). Vegetative propagation was maintained by subculture every 6-7 days by mechanical disruption of plant tissue using a tissue disruptor (TissueRuptor; Quiagen, Venlo, Netherlands) leading to predominant growth of chloronema. For gametangia and sporophyte development, protonema was cultured on sterile peat pellets (Jiffy7; Jiffy Products, Hoek van Holland, Netherlands) in plant culture boxes during 28 days, until gametophores were fully mature. Water was supplied to the bottom of each box containing 4 pellets and samples were transferred to $17^{\circ} \mathrm{C}, 8 \mathrm{~h}$ light and $50 \%$ humidity (light intensity $80-85 \mu \mathrm{mol} \mathrm{m}-2 \mathrm{~s}-1$ ) to induce the development of reproductive structures ${ }^{25}$. Further development of the sporophyte was conducted under these conditions.

Protoplast preparation. Protoplasts were prepared from protonema grown over 4 days in solid KNOPS media. We $\sim 200 \mu \mathrm{L}$ of fresh protonema were harvested and incubated for $30 \mathrm{~min}$ at $20^{\circ} \mathrm{C}$ in a solution of $500 \mathrm{mM} \mathrm{D}$-Mannitol plus $1 \%$ driselase (Sigma-Aldrich, St. Louis, MO, USA) that was centrifuged for $5 \mathrm{~min}(10,000 \mathrm{~g}$ ) Protoplasts were filtered ( $50 \mu \mathrm{m}$ mesh), pelleted ( 5 min centrifuge at $250 \mathrm{~g}$ ) and washed twice in $500 \mathrm{mM}$ D-Mannitol. They were finally transferred to the bath solution for patch-clamp (see below), stored in the dark at $4^{\circ} \mathrm{C}$ and studied by the patch-clamp technique within the next 3 hours. Protoplasts were selected under the microscope (20x magnification).

COS cell's transfection. COS-7 cells (provided at ATCC, Manassas, VA, USA) were used in low passage $(P<10)$. The $P p G L R 1$ coding sequence was introduced into pCI (Promega, Madison, WI, USA). COS cells were plated at a density of $50 \%$ confluence in 35-mm-diameter dishes and immediately transfected using FugeneHD (Promega, Madison, WI, USA) as specified by the supplier. pIRES-CD8 was co-transfected with $p C I-P p G L R 1$ to select transfected cells ${ }^{26}$. pIRES-CD8 is a pCI-derived vector that allows co-expression of the inserted sequence and of CD8 protein. For the transfection mix, we used $7 \mu \mathrm{L}$ of FugeneHD, $1.5 \mu \mathrm{g}$ of pCI-PpGLR1 and $0.2 \mu \mathrm{g}$ of pIRES-CD8 in $150 \mu \mathrm{L}$ water. Cells were transferred to new petri dishes 24 hours after transfection (by trypsin treatment), at low density for patch-clamp study. Cells were analyzed 36 to 48 hours after transfection. Transfected cells were detected with the anti-CD8 antibody-coated bead method (Dynabeads CD8, thermofisher) ${ }^{27}$.

Patch-clamp experiments. Pipettes were pulled with P97 puller (Sutter Instrument, Novato, CA, USA). Their resistance was: 3-5 Mohm for COS cells, and $15 \mathrm{Mohm}$ for protoplasts in the respective bath solutions. Currents were recorded after establishing the whole-cell configuration, filtered at $1-2 \mathrm{kHz}$ with a sampling frequency of $2-4 \mathrm{kHz}$ using an Axopatch 200A amplifier, a DigiData 1322A AD-converter (Molecular Devices, Sunnyvale, CA, USA) and WinWCP software (http://spider.science.strath.ac.uk/sipbs/software_ses.htm). $\mathrm{Na}_{2}$ 6-cyano-7-nitroquinoxaline-2,3-dione (CNQX, Santa Cruz Biotech, Dallas,TX USA) $(100 \mu \mathrm{M})$ was eventually added to the bath solution. For whole-cell recordings, the pipette solution contained $140 \mathrm{mM} \mathrm{NaCl}, 3 \mathrm{mM} \mathrm{MgCl}_{2}, 5 \mathrm{mM} \mathrm{EGTA}$, and $10 \mathrm{mM}$ Bis-tris propane $\mathrm{pH} 7.2$ (Hepes). For COS cells, the bath solution contained $10 \mathrm{mM} \mathrm{NaCl}, 130 \mathrm{mM} \mathrm{N}$-methyl-d-glucamine - $\mathrm{Cl}, 5 \mathrm{mM} \mathrm{CaCl}_{2}$, and $10 \mathrm{mM}$ bistris propane, pH 6.0 (MES). For protoplasts: $10 \mathrm{mM} \mathrm{NaCl}, 100 \mathrm{mM} \mathrm{N}$-methyld-glucamine - $\mathrm{Cl}, 20 \mathrm{mM} \mathrm{CaCl}_{2}$, and $10 \mathrm{mM}$ bis-tris propane, $\mathrm{pH} 6.0$ (MES). For protoplasts recordings, bath and pipette solutions were adjusted to $500 \mathrm{mosmol}$. kg-1 with D-mannitol. Voltage protocol: $1.5 \mathrm{~s}$ pulses from -100 or $-140 \mathrm{mV}$ to +40 or $+60 \mathrm{mV}(20 \mathrm{mV}$ steps $)$, holding potential $0 \mathrm{mV}$. Alternative internal solution for COS cells (Fig4G,H ) consisted of 10mM HEPES, $3 \mathrm{mM} \mathrm{MgCl}_{2}, 5 \mathrm{mM}$ EGTA and $1 \mathrm{mM} \mathrm{CaCl}_{2}$ for a final free $\mathrm{Ca}^{2+}$ concentration $<100 \mathrm{nM}$ (http://web. stanford.edu/ $\sim$ cpatton/webmaxcS). Pipette solutions were adjusted to $\mathrm{pH} 7.3$ with bis-tris propane and to 350 mosmol. $\mathrm{kg}^{-1}$ with $\mathrm{D}$-mannitol. Alternative external solutions for COS cell experiments were 1.) $3 \mathrm{mM} \mathrm{CaCl}_{2}$ and 2.) Calcium D-gluconate $7 \mathrm{mM}, 27 \mathrm{mM}$, or $67 \mathrm{mM}$ with $3 \mathrm{mM} \mathrm{CaCl}$. External solutions also contained $10 \mathrm{mM}$ bis-tris propane and adjusted to $\mathrm{pH} 7.3$ with HEPES and 350 mosmol. $\mathrm{kg}^{-1}$ with D-mannitol. Liquid junction potentials were corrected offline with Clampex 10.0.

$\mathrm{Ca}^{2+}$ imaging in COS-7 cells. COS-7 cells were co-transfected with $0.5 \mu \mathrm{g}$ pEF1-YC3.6 plus $1 \mu \mathrm{g}$ pCI-PpGLR1 as described above. Cells were detached by trypsin and transferred the day before imaging in $35 \mathrm{~mm}$ petri dishes with $14 \mathrm{~mm}$ glass coverslip (MatTek, Ashland, MA, USA). Cells were washed three times with the following bath solution: EGTA (1 mM), BTP (10 mM) pH7.3 (HEPES), with or without $\mathrm{GdCl}_{3}(1 \mathrm{mM})$ adjusted to 350 mosmol with D-Mannitol. In a first step, images were acquired with $500 \mu \mathrm{L}$ bath solution. After $2.5 \mathrm{~min}, 500 \mu \mathrm{L}$ of a solution containing $\mathrm{CaCl}_{2}(30 \mathrm{mM})$, BTP $(10 \mathrm{mM})$ pH7.3 (HEPES), with or without $\mathrm{GdCl}_{3}(1 \mathrm{mM})$ adjusted to 350 mosmol with D-Mannitol was added to the dish
Cells expressing the YC3.6 were imaged at room temperature using a DeltaVision Elite Deconvolution/TIRF microscope system (Olympus inverted IX-71) under a $60 \mathrm{X}$ lens (1.2NA UPLSAPO water lens / WD $0.28 \mathrm{~mm}$ ). Xenon lamp from the DeltaVision system was used with CFP excitation filter (415-445nm). Two simultaneous emission records were captured, for CFP emission (455-485 nm) and for $\mathrm{cp}$-Venus emission $(520-550 \mathrm{~nm})$. To minimize bleaching, the laser was set to $2 \%$, cp-Venus and CFP imaging were recorded with 0.3 and 0.2 sec exposure time respectively. A time-lapse acquisition was performed with a sampling interval of $30 \mathrm{~s}$. Images were processed using ImageJ (http://imagej.net/Welcome). Ratios were obtained after background subtraction and signal clipping using the "Ratio-plus" plug-in for ImageJ. Signal of each channel were averaged in a circle in the middle of the cell (circle with 100 - 200 pixel diameter depending on the size of the cell). The $\mathrm{cp}$-Venus/CFP ratio was obtained by dividing the emission recorded for $\mathrm{cp}$-Venus $(520-550 \mathrm{~nm})$ by the one recorded for CFP $(455-485 \mathrm{~nm})$. No significant bleaching or drift or the ratio was observed our experimental conditions. For qualitative analysis, images were processed in ImageJ with the Gaussian blur filter set to a radius of 0.75 pixels. Authenticated COS-7 cell lines used in all experiments were purchased from ATCC (https://www.atcc.org). No further testing for mycoplasma was done in our laboratory after purchase.

Tissue Isolation for RNA extraction. Triplicates for the different tissue samples were isolated from wild-type Gransden strain according with the particularities of each tissue. Archegonia were isolated from gametophores exposed to short day conditions for 15 days. Dissection was done manually under the stereoscope (SMZ800; Nikon, Tokyo, Japan) and placed into Trizol. At this point maturity was ensured and only closed organs were selected. 300 archegonia were used per replicate to isolate RNA. Sporophytes from different developmental stages were manually collected. Included stages were designated as S2, S3 and SM as defined previously ${ }^{9}$. Gametophores at the specific time points described above were dissected under the stereoscope and only the sporophytes fitting the desired morphological stage were isolated. At stage S2 the separation from archegonia tissue is not possible with our isolation methods and in order to maintain the comparative capabilities of our set, the residual archegonium was attached to all sporophytes collected. Samples were stored directly in Trizol. Approximately 25 sporophytes from each developmental stage were used for RNA extraction.

Caulonema development was induced by growing protonema under dark conditions in vertical KNOPS plates for 5 days, while chloronema was produced under normal conditions (16h light/8h dark). Both filaments were cut manually under a stereoscope (SMZ800; Nikon, Tokyo, Japan) and identity was confirmed by looking at the cell division plates. Tissue was directly placed in Trizol until RNA extraction. Rhizoids were harvested from gametophores grown in Magenta boxes (Sigma-Aldrich Co, St. Louis, US) containing KNOPS minimal medium ${ }^{24}$ under normal conditions ( $16 \mathrm{~h}$ light/8h dark). Around 100 gametophores per replicate were isolated and the entire rhizoids were manually dissected with a razor blade under a stereomicroscope (SMZ800; Nikon, Tokyo, Japan). Isolated rhizoids were frozen in liquid nitrogen right after dissection.

Gametophores grown on sterile peat pellets (Jiffy-7; Jiffy Products, Hoek van Holland, Netherlands) for 3 weeks were isolated manually and placed on Trizol for subsequent RNA extraction.

For sperm cell isolation, mature antheridia were manually dissected from gametophores at day 15 after induction. More than 50 Antheridia per preparation were placed on a cover slip with $50 \mu \mathrm{l}$ of sterile MilliQ water, where sperm release occurred naturally after few minutes. Using micromanipulators (CellTram; Eppendrof, Hamburg, Germany) attached to an inverted microscope (DMIRE2; Leica Microsystems, Wetzlar, Germany) the clusters of cells were collected with microcapillaries. The collected sperm was placed directly into Trizol, frozen in liquid nitrogen and stored at $-80^{\circ} \mathrm{C}$ until use. For RNA extraction, 200-400 clusters were used per replicate and four replicates were generated.

RNA purification and cDNA synthesis. RNA from all samples was isolated and purified using columns (Direct-Zol; Zymo Research, Irvine, CA) following manufacturer's instructions. Samples were treated with TURBO DNase (Ambion, Life Technologies, Waltham, US) at $37^{\circ} \mathrm{C}$ for $30 \mathrm{~min}$. RNA integrity and quantity were assessed on a Bioanalyzer using a 6000 Pico Assay (Agilent 2100; Agilent Technologies, Santa Clara, US). On average $7.5 \mathrm{ng}$ of RNA were used to synthetize cDNA using an amplification kit (Ovation Pico WTA System V2; NuGen Technologies, Leek, The Netherlands). The cDNA concentration obtained from all samples was roughly in the same range (180-260 ng/ $\mu \mathrm{l})$ and further quality controls were performed using a Bioanalyzer.

Determination of $P \boldsymbol{p} G L R s$ expression leves by qRT-PCR. cDNA was synthetized using RNA isolated from individual Physcomitrella tissues as described before. $1 \mathrm{ng}$ of cDNA was used per reaction, $10 \mu \mathrm{l}$ of enzyme mix (SYBR Green supermix; Bio Rad, Hercules, US), $5 \mu \mathrm{l}$ of each primer solution at $0.3 \mu \mathrm{M}$ and $5 \mu \mathrm{l}$ of diluted DNA. Each plate contained three technical replicates of every sample for each set of primers. We used the following primers for amplification qCR001 / qCR002 for 
PpGLR1; qCR003 / qCR004 for PpGLR2 and qCR005 / qCR006 for alpha tubulin (Supplementary Table 1). The thermal cycling protocol was as follows: initial $50^{\circ} \mathrm{C}$ for $2 \mathrm{~min}$, denaturation for $10 \mathrm{~min}$ at $95^{\circ} \mathrm{C}$ followed by 40 cycles of $30 \mathrm{~s}$ at $95^{\circ} \mathrm{C}$, $1 \mathrm{~min}$ at $59^{\circ} \mathrm{C}$ and $30 \mathrm{~s} \mathrm{~s}$ at $72^{\circ} \mathrm{C}$. We obtained Ct values using the program SDS 2.4 or with Bio-Rad CFX Manager with default threshold settings. Experiments were carried out in an ABI7900HT system (Applied Biosystems).

Constructs and Physcomitrella patens transformation. PpGLRs knock out mutants were generated by homologous recombination using the G418 and Hygromycin resistance cassettes to replace the gene of interest. Genomic sequences of approximately $600 \mathrm{bp}$ upstream and downstream of PpGLR1 (Pp1s56_31V6) and PpGLR2 (Pp1s253_26 V6) coding regions were amplified by PCR. To generate the plasmid used to replace PpGLR1 genomic coding sequence, we amplified the flanking regions of the gene with the primers oCR003 / oCR004 (upstream region), and oCR005 / oCR06 (downstream region). Amplified flanking fragments were cloned into pBNRF vector flanking the G418 cassette using SalI and BamHI, and SpeI and AscI for upstream and downstream regions respectively. Plasmid was linearized to transform Physcomitrella protoplasts. Two rounds of antibiotic selection were done: during the first round, regenerated protoplasts were transferred to KNOPS medium with geneticin (g418), resistant colonies were transferred back to medium without antibiotics and grown for further 10 days before going to the second 10 day antibiotic selection. After final selection, DNA was extracted from stable resistant colonies and used for PCR amplification reactions of the PpGLR1 gene. Colonies in which the presence of the genes could not be detected were selected as being putative K.O's and confirmed for the correct insertion of the resistance cassette by further PCR experiments (Extended Data Fig. 7 a, c). Three independent knock out colonies were generated for Ppglr1 (Ppglr1-19, Ppglr1-8 and Ppgl1-14).

To generate Ppglr1/2 double knock, we amplified the flanking regions of PpGLR2 using the primers oCR015 / oCR016 and oCR017 / oCR018 for upstream and downstream regions respectively, and cloned them into the pBHrev plasmid flanking the hygromicin resistance cassette. Cloning was done using AscI and SpeI (upstream fragment), and $\mathrm{SmaI}$ and $\mathrm{XbaI}$ (downstream fragment) restriction enzymes. Plasmid was linearized and used to transform protoplasts obtained from the Ppglr1-19 mutant line. Selection and genotyping of stable mutants was done as before (Extended Data Fig 7. b, d). For detection of $P p G L R 1$ gene we used oCR001 / oCR002 primer pair and for PpGLR2 gene we used oCR013 / oCR014 primer pair. Three independent double knock out colonies were generated (Ppglr1/2-63, Ppglr1/2-11 and Ppglr1/2-16) (Extended Data Fig 7.b, d). Confirmed independent mutant lines for single and double knock out mutants were phenotyped (quantification of sporophyte production) confirming that deletion of $P p G L R s$ had a similar effect in all lines with the same mutant background (Extended Data Fig. 8 a, b). The phenotypes presented in the result section correspond to Ppglr1-19 single knock out and to $P p g l r 1 / 2-11$ double knock out lines, except for microarray experiments in which Ppglr1/2-63 was used.

Constitutively over expressing PpGLR1 line (35s::PPGLR1) was generated by amplifying the $P p G L R 1$ coding region from protonema $C D N A$ with the primer pair oCR023 / oCR024 and cloning this region into the Gateway pDONR 221 vector (Invitrogen). PpGLR1 coding region was then subcloned into the p108GW35s plasmid, which carries the 108 locus of Physcomitrella, by LR reaction using an enzyme mix (Gateway LR Clonase II enzyme mix; Invitrogen, Waltham, US). The resulting vector was used for protoplast transformation and geneticin resistant colonies were selected as before. Overexpression of $P p G L R 1$ in stable colonies was confirmed by RT-PCR using the primers oCR001 / oCR002 and oCR044 / oCR045 for PpGLR1 and alfa tubulin amplification respectively (Extended Data Fig. 9 e). The 35s::PpGLR1-2 line was used for subsequent experiments.

Generation of the Ppglr1/2 line constitutively expressing GFP (Ppglr1/2 GFP) was done by crossing the double knock out line with a transgenic line overexpressing GFP under the 2x35s promoter (gift by Pierre Perroud, Washington University in St Louis, St Louis, USA). After crossing these lines, hybrid sporophytes produced by Ppglr1/2 were identified under a fluorescent stereo microscope (Zeiss Stero Lumar; Jena, Germany) and collected. Spores obtained from hybrid sporophytes were germinated resulting in protonemal colonies. Those colonies positive for GFP fluorescence were selected and genotyped. We used primer pair oCR001 / oCR002 and oCR 013 / oCR014 for amplifying PpGLR1 and PpGLR2 genes respectively, and further selected the colonies lacking both genes. We obtained four Ppglr1/2 GFP independent lines, and only used the colony with the highest fluorescence intensity for performing experiments.

A Ppglr2 single knock out line in which the PpGLR2 coding sequence was replaced by the $\beta$-glucuronidase reporter gene (GUS) was generated by homologous recombination. The flanking regions $(\approx 700 \mathrm{bp})$ of $P p G L R 2$ were amplified with primers oCR034 / oCR035 and oCR036 / oCR037 for upstream and downstream regions respectively. Both fragments were cloned into the pTN83 plasmid flanking the GUS gene, the nopaline synthase polyadenylation signal (Tnos) and the geneticin resistant cassette using NotI and BamHI restriction enzymes (upstream flanking fragment) and EcoRI and SalI (downstream fragment). Plasmids were linearized and protoplast transformation, selection, and genotyping was done as before (Extended Data Fig. 9 a, c).

PpGLR2::BELL1 complementation line was generated by homologous recombination using the Ppglr1/2 knock out background. A construct containing the PPBELL1 coding sequence followed by the Tnos terminator sequence and the zeocin resistant cassette was inserted in situ immediately downstream of the PPGLR2 promoter, which resulted in the replacement of the genomic region containing the hygromicin resistant cassette. The vector used for transformation, pZB1, was generated as follows: The coding region of PpBELL1 was amplified by PCR from mature sporophyte cDNA with the primers oCR025/oCR026 and cloned into pBNRF plasmid using XhoI and SalI restriction enzymes. Subsequently, the upstream flanking genomic region of $P p G L R 2$ gene was amplified using the primers oCR029 / oCR030 and cloned into the same plasmid using AvrI and SalI. A fragment containing the Tnos terminator followed by the zeocin resistance cassette was amplified from the pTFH38.9C plasmid with the primers OCR032 / oCR033 and cloned into pBNRF using XhoI and ApaI, replacing in this way the G418 resistance cassette. The resulted plasmid was renamed as pZB1. Finally, the downstream flanking genomic region of $P p G L R 2$ was amplified with the primers oCR010 / oCR011 and cloned into pZB1 using ApaI and AscI (Extended Data Fig. 9 b) Plasmid was linearized for the transformation of Ppglr1/2-11 double knock protoplasts. Selection of zeocin resistant colonies was done as before and stable colonies were genotyped to confirm correct insertion (Extended Data Fig. 9 d). Three independent lines were obtained (PpGLR2::BELL1-18, PpGLR2::BELL1-57 and $P p G L R 2:: B E L L 1-10$ ). All lines were analyzed showing similar phenotypes (Extended Data Fig $8 c$, d). Results presented in the main text were obtained using PpGLR2::BELL1-18

Assessment of protonema growth under different $\left[\mathrm{Ca}^{2+}\right]$. For determining protonema growth, small pieces (around $1 \mathrm{~mm}$ in diameter) of recently grinded tissue ( 5 days after the last grinding propagation) were plated in modified KNOPS medium supplemented with different calcium concentrations. For the preparation of such medium the concentration of $\mathrm{Ca}\left(\mathrm{NO}_{3}\right)_{2}$ was reduced and substituted for $\mathrm{KNO}_{3}$ as a source of nitrogen. Standard used agar (Formedium agar; Foremedium, Hunstanton, UK) was also substituted by purified agar with low content of residual $\mathrm{Ca}^{2+}$ (Sigma A7921; Sigma-Aldrich, St. Luis, US). Pictures of protonema colonies were taken at day 0 and day 5 . Protonema growth was estimated as the $\Delta$ in colony diameter from day 0 to day 5 . Results are presented as averages in $\Delta$ colony diameters.

Measurements of caulonema length. For induction of caulonema filaments fresh protonema was grown for 7 days under long day conditions. After this period protonema was collected using a pair of forceps and then distributed forming horizontal lanes in a petri dish with KNOPS medium and a cellophane layer. The plate was covered with aluminium foil to create completely dark conditions and placed on a vertical position (caulonema is known to grow negatively to the gravity vector) at $25^{\circ} \mathrm{C}$ for 5 days. Finally, caulonema were measured longitudinally from the tip of the filament until the first branch at the base. Image J was used for calculating distance.

Quantification of sporophyte production rates. Induction of gametangia was conducted after 28 days of growth on sterile peat pellets (Jiffy-7; Jiffy Products, Hoek van Holland, Netherlands). The phenotyping of the lines was done once the sporophytes reached the mature stage ( 6 weeks after gametangia induction) Fertility rates (percentage of gametophores with sporophytes) was assessed by counting successfully developed sporophytes in at least 100 randomly collected gametophores per sample. Previous characterization of fertility rates in $w t$ showed that under our conditions sporophyte production rates were between $45-60 \%$. Furthermore, we observed that samples with lower production rates always showed defects like fungus or bacteria contamination, or low humidity. Therefore samples from both genotypes with fertility rates below $45 \%$ were discarded for statistica analysis, considered as stress affected. Statistical differences between samples were estimated using a Student's t-test or Wilcoxon-Mann-Whitney test as needed.

Cross-fertilization experiments. Protonema tissue from the two strains being crossed was collected in approximately equal amounts and placed on a glass container with water. Tissue was grinded with a tissue disrupter (TissueRuptor Qiagen, Venlo, Netherlands) and transfered to sterile peat pellets (Jiffy-7; Jiffy Products, Hoek van Holland, Netherlands) in plastic containers. Gametophores were grown and exposed to gametangia inducing conditions as described before. 15 days after transfer, approximately $30 \mathrm{ml}$ of tap water was added to the top of the gametophore mesh. This step was repeated 3 times to ensure water reached most of the gametophores. On the next day the procedure was repeated and excess water was decanted. Samples continued under gametangia inducing conditions for 3-4 weeks. After this period sporophytes were visualized under a fluorescent stereomicroscope (Zeiss Stereo Lumar; Jena, Germany). 
Visualization and collection of Physcomitrella reproductive organs. Reproductive organs (antheridia and archegonia) were manually isolated from gametophore apexes under a stereomicroscope using very fine forceps (Dumoxelbiology \#5; Electron Microscopy Sciences, Hatfield, US). For visualization samples were placed on a microscope slide with water and images were acquired on an upright microscope (Leica DM LB2; Leica Microsystems, Wetzlar, Germany), equipped with a colour camera (UI-3370CP Rev.2, IDS, Obersulm, Germany) using a 20X objective with bright field illumination. For RNA extraction, isolated reproductive organs were collected directly into trizol reagent. RNA purification for cDNA synthesis was done as described before and used for microarray hybridization.

Quantification of spore size and numbers. Spores were obtained by bursting mature sporophytes into sterile water. In the case of $w t, P p g l r 1$ and Ppglr2, the content of three mature sporophytes was released in $250 \mu \mathrm{l}$ of sterile water. Since Ppglr1/2 had significantly fewer spores, the content of three sporophytes was released in $100 \mu \mathrm{l}$ of water to keep adequate spore densities. For calculating the spore two-dimensional area, pictures were taken with an inverted microscope (Leica DMRA2, Leica Microsystems, Wetzlar, Germany) using a 20X objective with bright field illumination and measured using Image J "measuring" function (http://imagej.nih.gov/ij/). Determination of the number of spores per sporophyte was done by taking $10 \mu \mathrm{l}$ of the samples containing spores (prepared as mentioned before) and placing them on a haemocytometer chamber (NeuBauer Blaubrand, Sigma-Aldrich, St. Luis, US). Both upper and lowers grids were used for counting. Each sample was measured three times to minimize variation.

Microarray hybridization and data analysis. $750 \mathrm{ng}$ of cDNA was used for labelling and hybridization on custom Nimblegen $12 \times 135 \mathrm{~K}$ arrays (Roche NimbleGen, Madison, US) following manufacturer's instructions in the IRB Barcelona Functional Genomics Core Facility (FGC). Arrays were scanned and raw data was obtained using the DEVA software, applying Robust Multichip Average (RMA) normalization to all arrays (Roche NimbleGen, Madison, US). Differential expression analysis was conducted using dChip software ${ }^{28}$ (http://www.hsph.harvard. edu/cli/complab/dchip/). Data was imported as External Data in tab-delimited text format, including expression data and standard error. In order to determine differentially expressed genes pair-wise comparisons between Ppglr1/2 and wt samples were conducted. For obtaining the list of down regulated genes in $P p \operatorname{glr} 1 / 2$ reproductive organs with respect to $w t$ we used a lower-confidence bound fold-change (LCB FC) cut-off of 3 . A total of 11 genes were obtained using this stringent cut-off. Information about gene function was obtained from the P. patens annotation v1.6 release 2012.3 (cosmoss.org), combined with information from several alignments against non-plant organisms and STRING information. Raw and analysed data can be obtained from array express database (https://www.ebi.ac.uk/arrayexpress/), accession number E-MTAB-5268.

Determination of sperm cell swimming behaviour. For quantifying the number of sperm cells that successfully target the archegonia entrance, reproductive units were isolated from gametophores exposed to gametangia inducing conditions for 15 days. One intact reproductive unit was isolated (the entire set of archegonia and antheridia produced at the tip of one gametophore) and placed in a $35 \mathrm{~mm}$ glass bottom dish (Nunc, Thermo Fisher, Waltham, USA) containing $60 \mu \mathrm{l}$ of sperm medium composed of $0.45 \mathrm{mM} \mathrm{CaCl} 2,0.3 \mathrm{mM} \mathrm{MgSO} 4,0.02 \mathrm{mM} \mathrm{KNO} 3$ and 0.081 $\mathrm{NaHCO}$. After sperm cells were released from the antheridia (typically one cluster of sperm cells per sample was released) the archegonia entrance was monitored for $45 \mathrm{~min}$ and time lapse image acquisition was done every time a sperm cell was spotted swimming close to the archegonium. Monitoring of the sample and time lapse images were acquired on a High Content Screening (HCS) inverted microscope (Leica HCS; Leica Microsystems, Wetzlar, Germany) equipped with a sCMOS camera (Flash Orca LT 4.0; Hamamatsu, Japan) at 80 frames per second, and using a $20 \mathrm{X}$ objective with bright field and DIC (differential interference contrast) illumination.

For determining differences in sperm cells' swimming behaviour, reproductive units were isolated and placed under the same conditions as before. Time-lapse imaging was done after the sperm cells manage to free themselves from the protein matrix and started swimming freely in the medium. Tracking of their two dimensional paths and calculation of different swimming parameters was done using the "manual tracking" plugin that comes included in the Image J software package.

Measurements of cytoplasmic $\left[\mathrm{Ca}^{2+}\right]$ in sperm cells. Sperm cells were incubated in $60 \mu \mathrm{l}$ of sperm medium (composed of $0.45 \mathrm{mM} \mathrm{CaCl} 2,0.3 \mathrm{mM} \mathrm{MgSO} 4,0.02 \mathrm{mM}$ $\mathrm{KNO} 3$ and $0.081 \mathrm{NaHCO} 3$ ) containing $10 \mu \mathrm{M}$ Fluo-4-AM for 1 hour. Medium also contained a high number of archegonia. Images were acquired on a High Content Screening (HCS) inverted microscope (Leica HCS; Leica Microsystems, Wetzlar, Germany) equipped with a sCMOS camera (Flash Orca LT 4.0; Hamamatsu, Japan), under a 20X/NA1.2 water immersion objective. Fluorescent images were acquired with a $488 \mathrm{~nm}$ excitation wavelength and 525/36 emission filter. Image analysis and quantification followed protocols and principles established elsewhere ${ }^{45}$. In short, fluorescence profiles were manually extracted using line segments to average across 7 pixels width using Image,, including the neighbouring background. Cells outside of regions prone to artefacts and distortions were selected based on a minimum visible fluorescence from two independent inductions per genotype. Local polynomial fits with different coarseness were performed in the raw fluorescence profiles in order to estimate the background and subtract it from the signal. Fluorescence peaks of individual cells were extracted from the fine-grained smoothing having the background, estimated from the coarse-grained smoothing, subtracted to yield the normalized $\mathrm{Ca}^{2+}$ signal.

Gene network analysis. Gene network analysis of $P p G L R 1$ and $P p G L R 2$ was done using the PlaNET tool (http://aranet.mpimp-golm.mpg.de/index.html). Networks were constructed using the HCCA algorithm with expression data for Physcomitrella patens available in the PlaNET database (default parameters). Each network is composed by genes located two steps away from the query gene. Functional enrichment analysis was done using the DAVID Bioinformatics Resources $6.7^{29}$ (https://david.ncifcrf.gov) based on A. thaliana homolog genes. Genes were clustered according to their GO terms, including biological process, molecular function, and cellular component. Enrichment scores were calculated based on the EASE scores (modified Fisher exact $P$ value) of each cluster term members. Default parameters were used for the analysis.

Statistics. Minimal sample size estimation was calculated using the Soper D. Statistics Calculators version 4.0 (http://www.danielsoper.com/statcalc/default. aspx) to ensure a statistical power level of at least 0.7 , a Cohen's $d$ of 0.5 , and a probability of 0.05 for a two-tailed t-test. Technical challenges of tracking sperm cell mobility and determining electrophysiological properties of PpGLRs imposed sample size restrictions; therefore, sample size estimation was not calculated for experiments shown in Figs. 2 and 4. However we estimated the effect size taking into account the mean and standard deviation of samples used for these experiments using the Ellis PD Effect size calculators (http://www.polyu.edu.hk/mm/ effectsizefaqs/calculator/calculator.html). Effect size was at least 0.8 (Cohen's d) for all comparisons shown in Fig. 2, which is considered a large effect.

Unpaired sample $t$-tests were used to compare $w t$ vs single or double knock out mutant samples. In all cases only two groups were compared at a single time. Variance, normality, and $p$ values were calculated using the Sigma Plot 11 software package (https://systatsoftware.com/products/sigmaplot/). Unequal variances approach was applied as necessary by the software. In cases were data was not normally distributed the non-parametric Mann-Whitney $U$ test was used. Alternatively the statistical programming language $\mathrm{R}$ was used to perform Welch's unequal variances $\mathrm{t}$-test, local polynomial fits and violin plots.

Data Availability. Microarray raw and normalized data can be accessed in the array express database (https://www.ebi.ac.uk/arrayexpress/), accession number E-MTAB-5268. Reprints and permission information is available at www.nature. $\mathrm{com} /$ reprints. Source data is provided for Figures 1d, 1c, 2b, 2c, 2d, 2e, 2f, 2i, 3b, 3c, 4d, 4f, 4h, and Extended Data Figure 1e 4b, 4d, 5b, 5d, 5f, 6b, 6d, 6f, 6h. Uncropped gels for gel images presented in Extended Data Figure 7 and 9 are provided as supplementary information. 
a

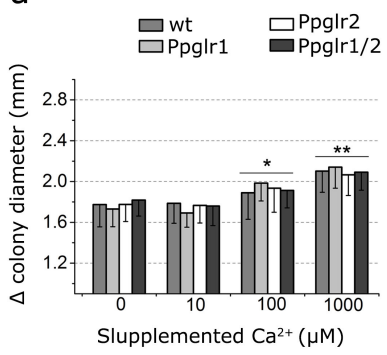

b

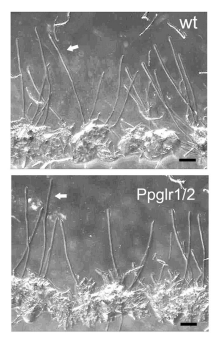

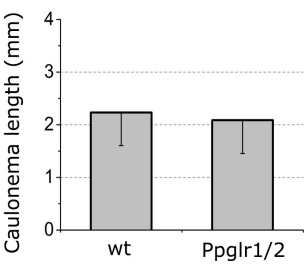

d

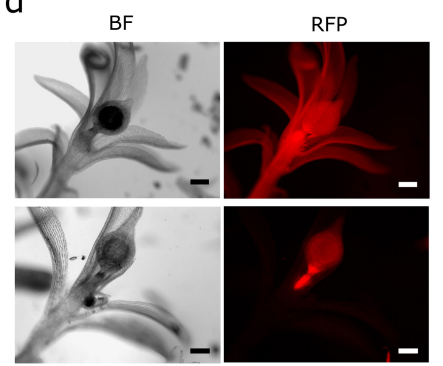

Extended Data Figure $1 \mid$ Measurement of caulonema filaments and cross-fertilization. (a) Growth of protonema colonies under different $\mathrm{Ca}^{2+}$ concentrations. Initial colony diameter was subtracted from diameter at day 5. Data from three biological replicates and several independent colony measurements. $n=59(w t), n=58$ (Ppglr1), $n=58($ Ppglr 2$) n=55$ (Ppglr1/2). Error bars represent the standard deviation from the mean. There was a statistically significant difference between $\mathrm{Ca}^{2+}$ treatments $(p<0.001, F 156.3)$, but not between genotypes $(p=0.952)$, determined by two-way ANOVA. Using Holm-Sidak multiple comparison we determined that colony diameter in samples treated with 100 and $1000 \mu \mathrm{M} \mathrm{Ca}^{2+}$ was significantly different from untreated samples $(p=0.01$ and $p<0.001$, single and double asterisks respectively). (b) Caulonema cells (white arrows) from $w t$ and Ppglr1/2 mutant have similar morphologies. Scale bars correspond to $500 \mu \mathrm{M}$ (representative of 13 images) (c) Caulonema

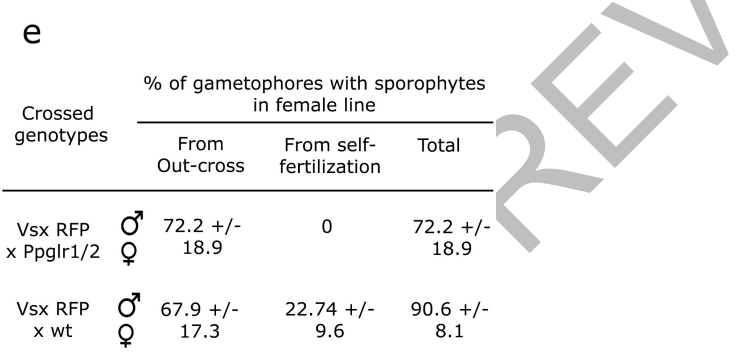

length from filaments induced in darkness was measured. There was no significant difference between $w t(n=148)$ and Ppglr1/2 $(n=180)$. Error bars represent the standard deviation from the mean. Results were obtained from three biological replicates. (d) Self-fertilization events (upper panels) and outcrossing events (lower panel) were identified in cross-fertilization experiments between Vsx RFP (parental line) and Ppglr1/2 (maternal line) (scales in panels correspond to $200 \mu \mathrm{m}$; representative of 4 images). (e) Sporophyte production rates in crossfertilization experiments using different genotypes are shown. Sporophyte production in Ppglr1/2 was as high as wt (outcrossing rates), suggesting full phenotypic complementation when $V s x$ sperm cells are provided. 50 gametophores per replicate were analysed. Mean \pm s.d. from 4 biological replicates. 
a

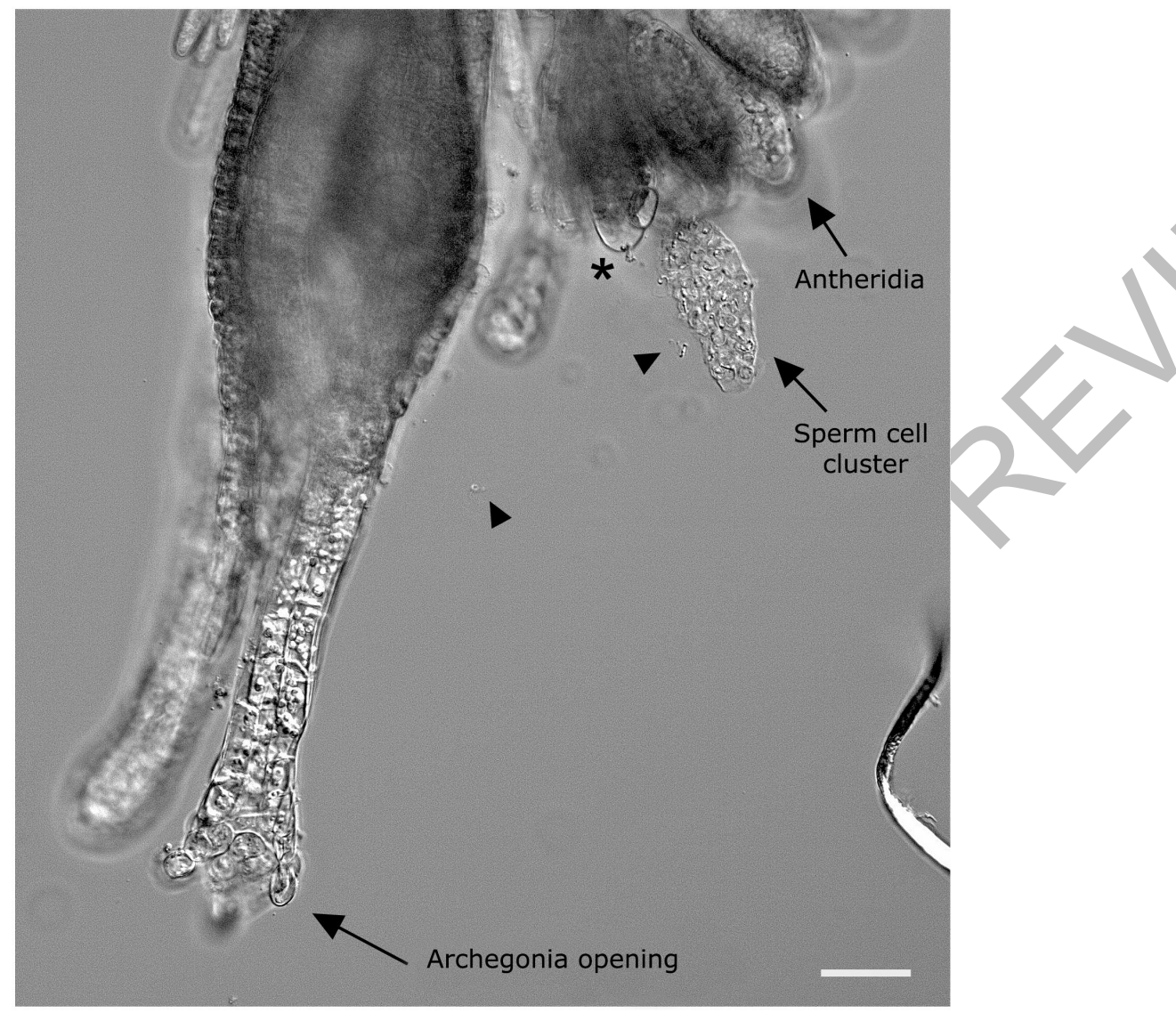

b

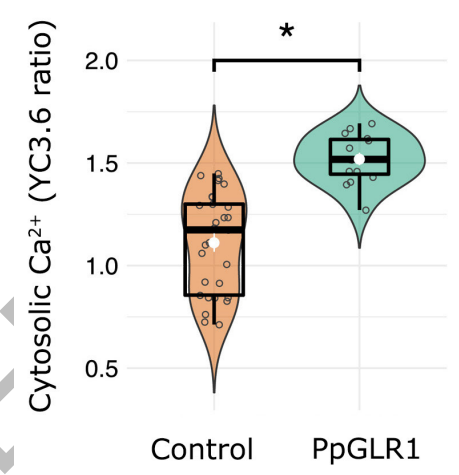

Extended Data Figure 2 | Display of an isolated reproductive unit, and quantification of cytosolic calcium and sporophyte production.

(a) An intact open archegonium, several antheridia, and a sperm cell cluster are shown (arrows). In most samples only one antheridium was ready to release the sperm cells at the time of the experiment. The antheridium from which the sperm cell cluster was released is indicated with an asterisk. After 1 minute, the released sperm cells became fully motile and individual cells began to separate from the protein matrix (arrowheads). Scale bar corresponds to $30 \mu \mathrm{m}$. Representative of 6 images. (b) Basal cytosolic calcium in control cells (transfected with $\mathrm{pCI}$ and pEF1-YC3.6) and cells co-expressing PpGLR1 and YC3.6 exposed to
C

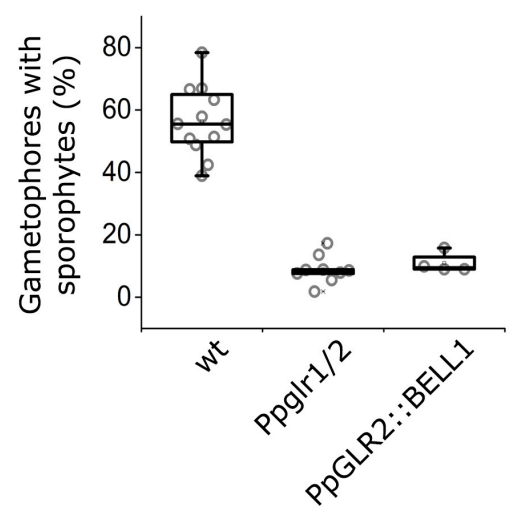

medium with no calcium. $\mathrm{n}=29$ (control), $\mathrm{n}=15$ (PpGLR1), biological replicates. There was a statistically significant difference between the two samples $\left(p=3 \times 10^{-8}\right)$ calculated with Welch's $t$-test for unequal variances. Violin plots show curves of density estimates, boxplots and white points with lines as mean and standard errors. (c) PpGLR2::BELL1 show similar sporophyte production rates as $P p g l r 1 / 2$. The percentage of gametophores with sporophytes from the total number of gametophores is shown.

Mean \pm s.d. from several independent biological replicates. $n=12(w t)$, $n=7$ (Ppglr1/2), $n=4$ (PpGLR2::BELL1). 100 gametophores were counted per sample. Box plot lines show outliers. 


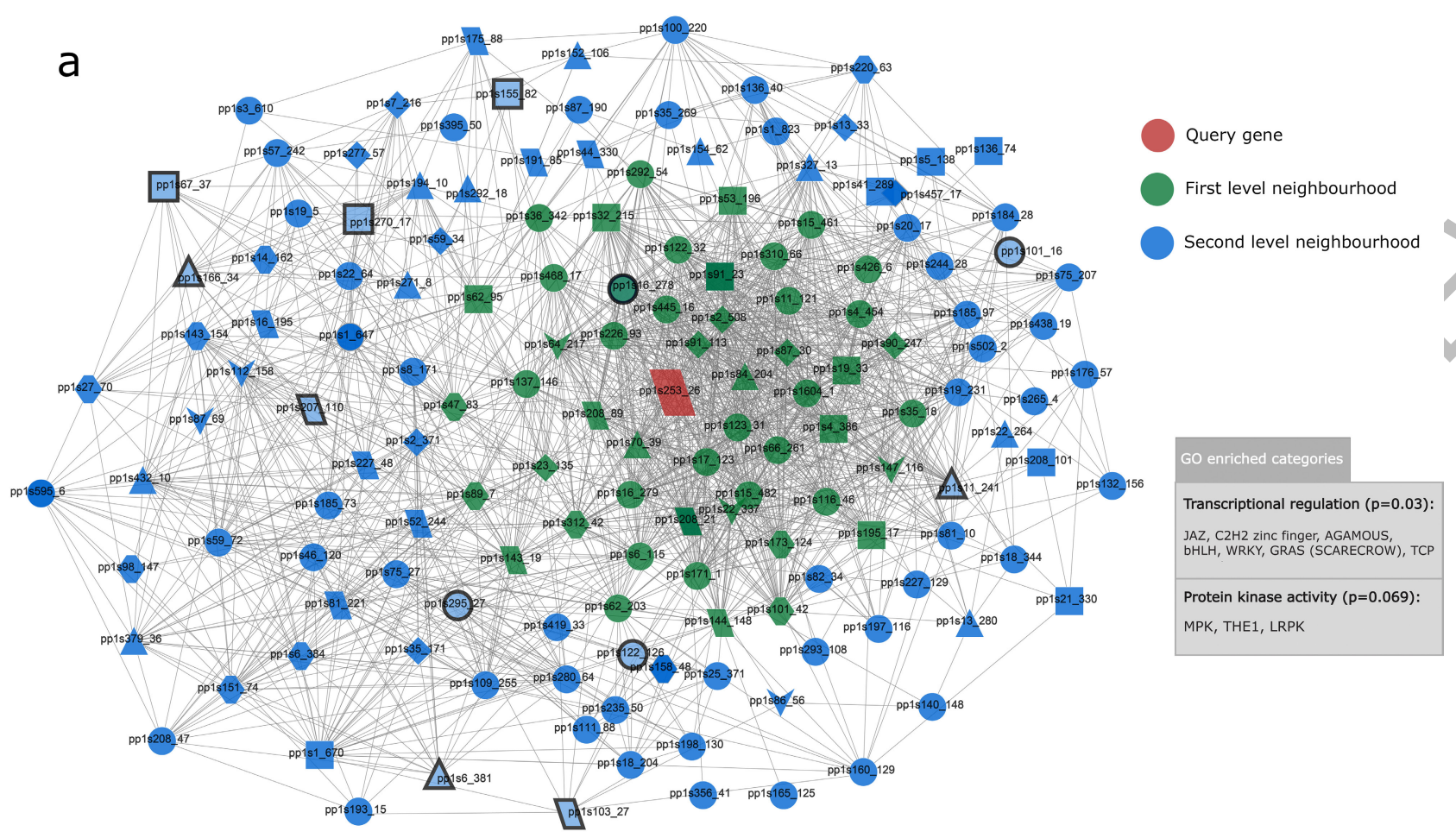

b

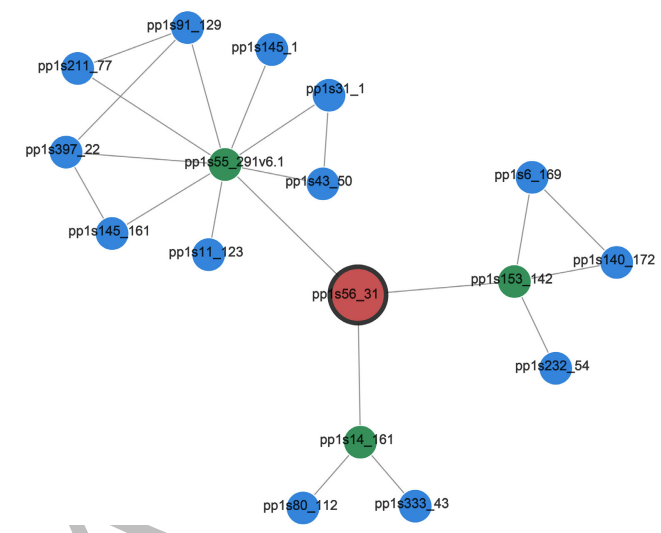

Extended Data Figure 3 Gene network analysis for PpGLR1 and PpGLR2. (a) $P p G L R 2$ co-expression gene network showing genes one and two steps away from the query gene. Gene ontology analysis of the 151 genes that integrate the network shows only two enriched categories: trancriptional regulation and protein kinase activity (modified Fisher exact $p$ value). The gene families represented in the two clusters are shown in italics. Highlighted nodes represent transcription factors genes interacting with PpGLR2 in the netwrok. (b) PpGLR1 co-expression gene network showing genes two steps away from the query gene. No enriched gene ontology categories were found. PpGLR1 is highlited at the center of the network. Shapes represent label co-ocurrances used to group genes according to Pfam domains and PLAZA families. For looking at the complete gene lists and annotation see Supplementary Table 3. 
a

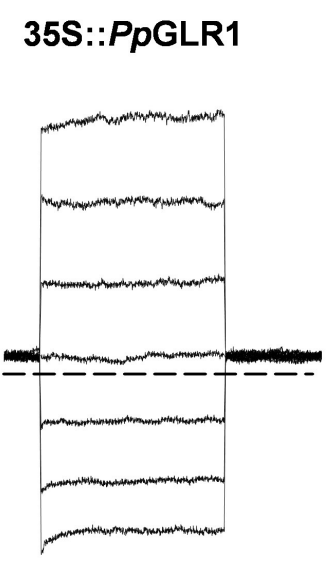

$$
\begin{gathered}
\text { 35S::PpGLR1 } \\
\mathrm{Gd}^{3+} 0.5 \mathrm{mM}
\end{gathered}
$$
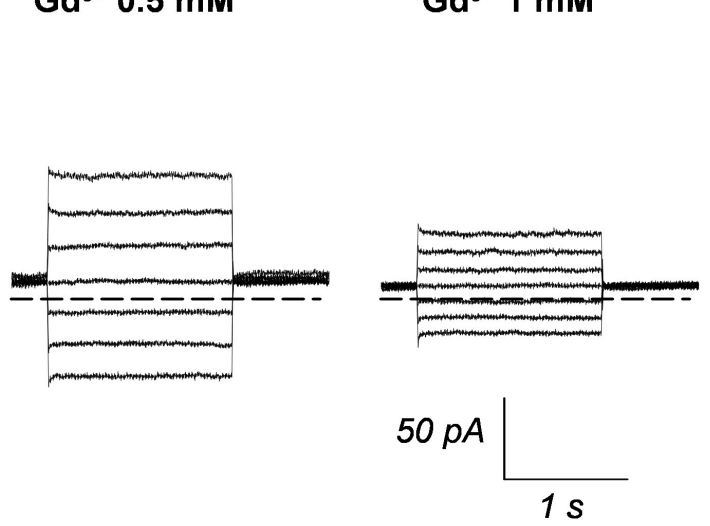

\section{S::PpGLR1 \\ $\mathrm{Gd}^{3+} 1 \mathrm{mM}$}

$1 \mathrm{~s}$

b

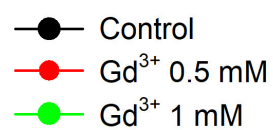

$\mathrm{Gd}^{3+} 1 \mathrm{mM}$

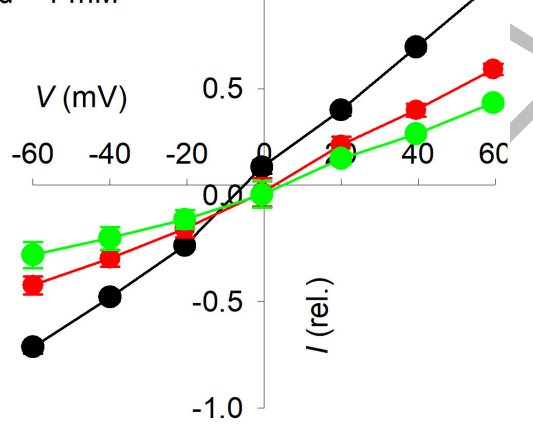

C

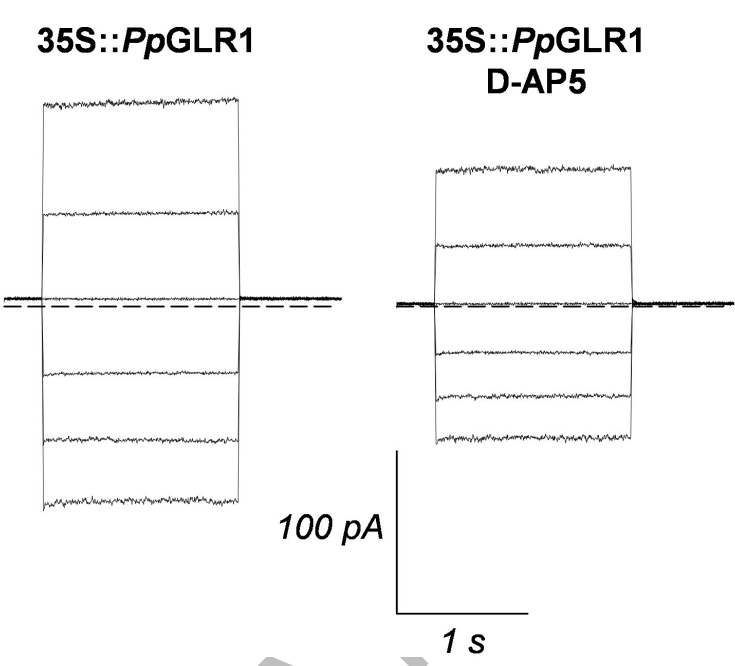

d

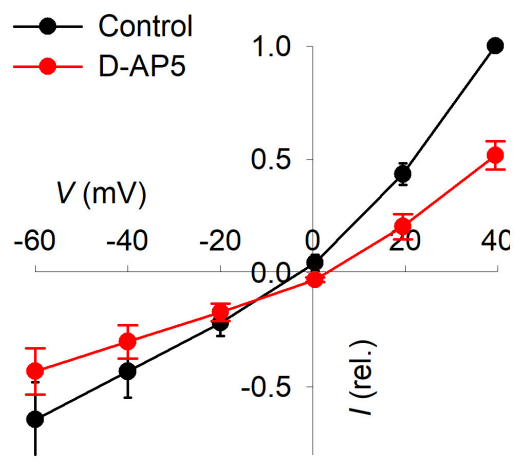

Extended Data Figure 4 | Effect of $\mathrm{Gd}^{3+}$ and D-AP5 on 35s::PpGLR1 protoplasts currents. (a) Typical currents recorded in protoplasts from 35 s::Pp GLR1 protonema under the whole-cell configuration before and after the application of 0.5 and $1 \mathrm{mM} \mathrm{Gd}^{3+}$. (b) Standardized current/ voltage curves of stationary currents recorded in experiments as shown in a. Black circles: control conditions, red circles: after the addition of $0.5 \mathrm{mM}$ $\mathrm{Gd}^{3+}$, green circles: after the addition of $1 \mathrm{mM} \mathrm{Gd}^{3+}(\mathrm{n}=3)$. Error bars represent the standard error of the mean. (c) Effect of D-AP5 $(200 \mu \mathrm{M})$ on typical currents recorded in protoplasts from 35S::PpGLR1 $(n=5)$. (d) Standardized currents corresponding to c., recorded before (black circles) and after (red circles) the addition of $200 \mu \mathrm{M}$ D-AP5 $(\mathrm{n}=4)$. In all cases $n$ represents biological replicates. Error bars represent the standard error of the mean. 
a

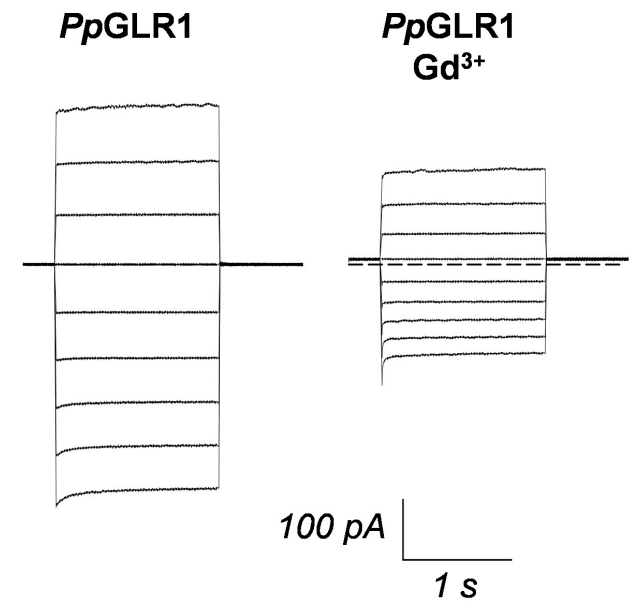

C

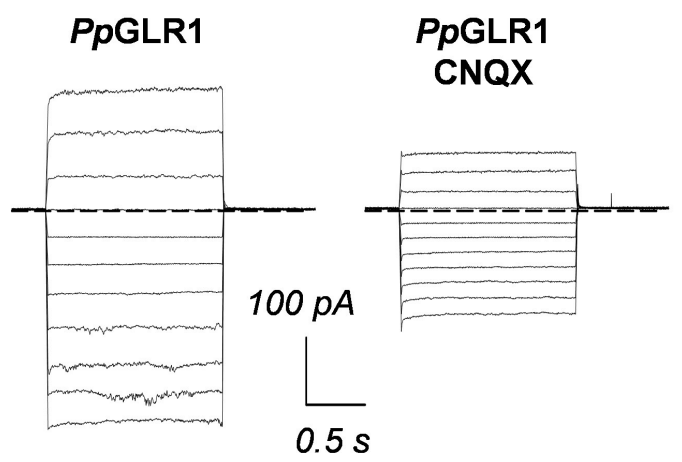

e

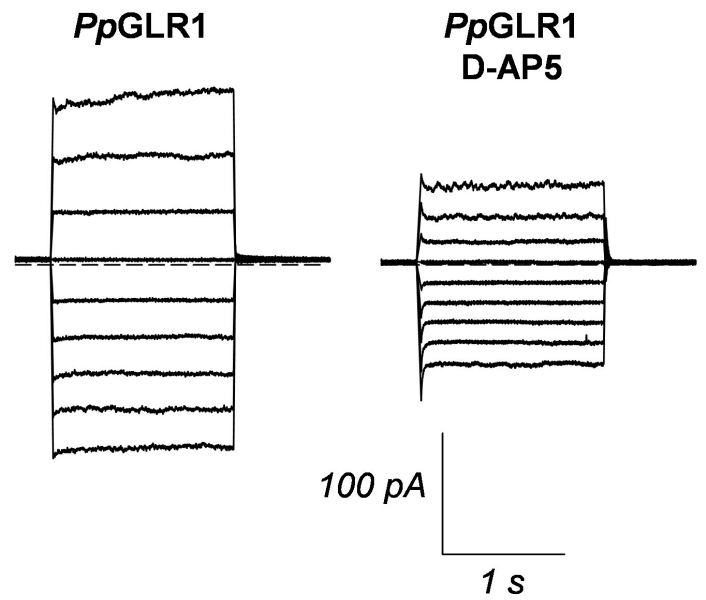

Extended Data Figure 5 | Effect of Gd ${ }^{3+}$, CNQX and D-AP5 on COS-7 cells expressing PpGLR1. (a) Typical currents recorded in COS-7 cells transfected with pCI-PpGLR1 before and after the addition of $\mathrm{Gd}^{3+}$ $(1 \mathrm{mM})$. (b) Standardized current/ voltage curves of experiments in a. Currents were recorded in control conditions (black circles), prior to $\mathrm{Gd}^{3+}$ application, and after the addition of $1 \mathrm{mM} \mathrm{Gd}^{3+}$ (red circles) $(n=3)$. (c) Typical currents recorded in transfected COS-7 cells expressing PpGLR1 in control solution (left) and after the addition of $50 \mu \mathrm{M}$ CNQX (right). (d) Standardized current/ voltage curves of experiments in c. Currents were recorded in control conditions (black circles), and after b

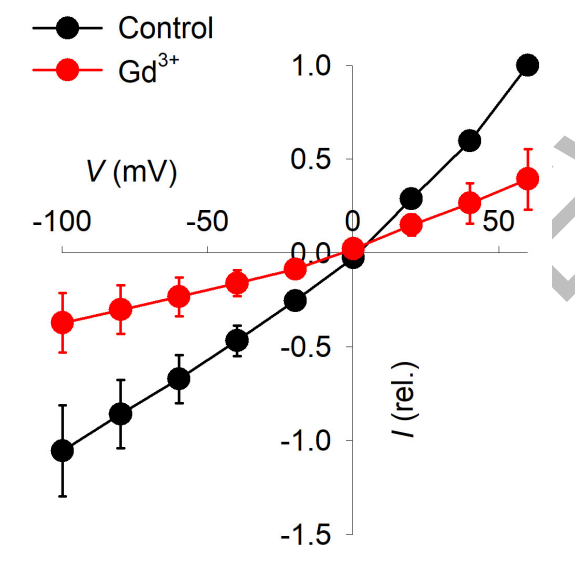

d

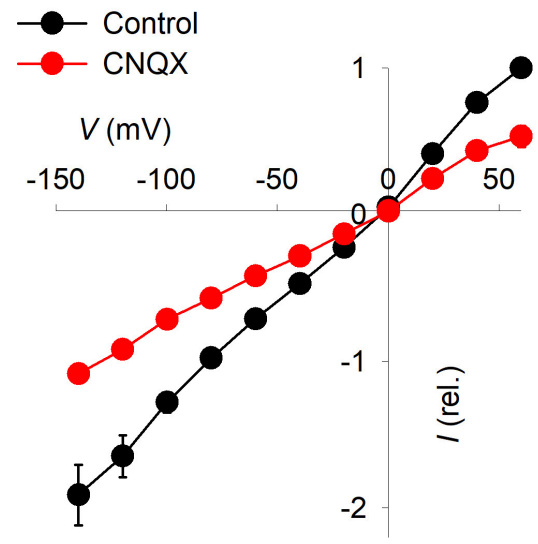

$f$

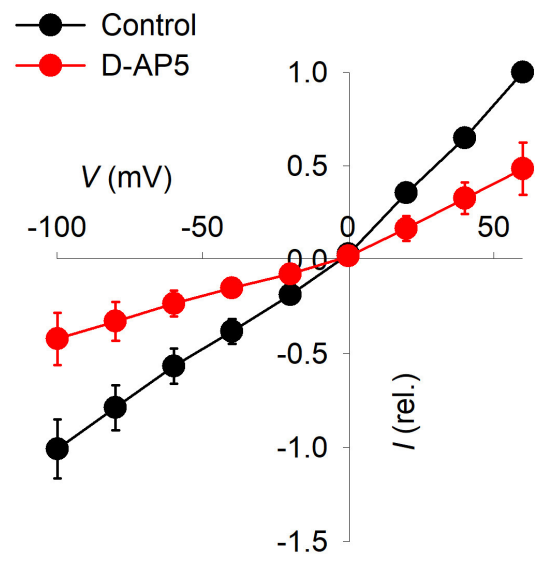

the addition of $50 \mu \mathrm{M}$ CNQX (red circles) $(n=3)$. (e) Typical currents recorded in COS-7 cells transfected with pCI-PpGLR1 before and after the addition of D-AP5 $(200 \mu \mathrm{M})$. (f) Standardized current/ voltage curves of experiments presented in e. Currents were recorded in control conditions (black circles) and after the addition of $200 \mu \mathrm{M}$ D-AP5 (red circles) $(n=4)$. Standard solutions are identical to Fig. 4E, F. Pipette contained $\mathrm{Cl}^{-} 146 \mathrm{mM}$ and $\mathrm{Na}^{+} 140 \mathrm{mM}$, bath solution contained $\mathrm{Cl}^{-} 150 \mathrm{mM}, \mathrm{Ca}^{2+} 5 \mathrm{mM}$ and $\mathrm{Na}^{+} 10 \mathrm{mM}$ as charge carriers. All error bars represent standard error. In all cases $n$ represents biological replicates. 


\section{RESEARCH LETTER}

a

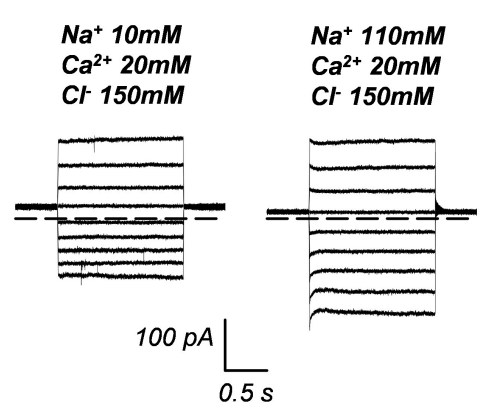

C
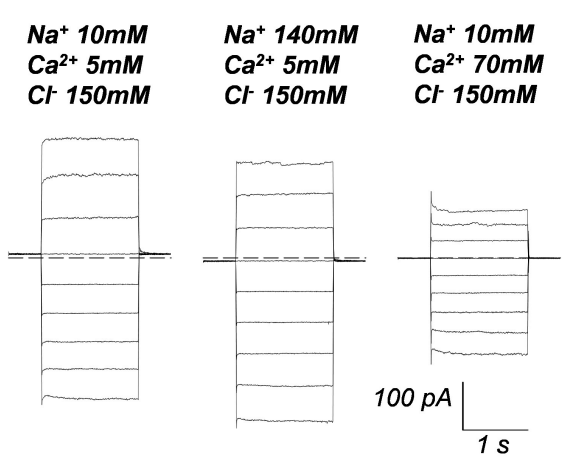

e

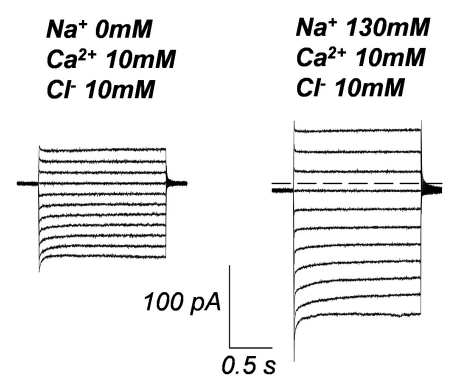

g

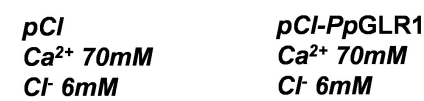

$$
\mathrm{Cr} 6 \mathrm{mM}
$$
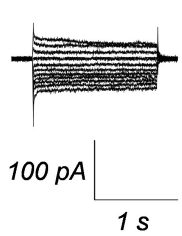

b

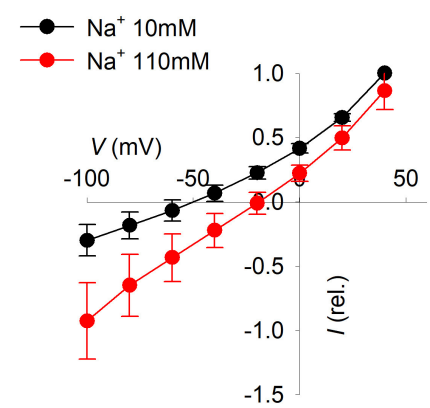

d

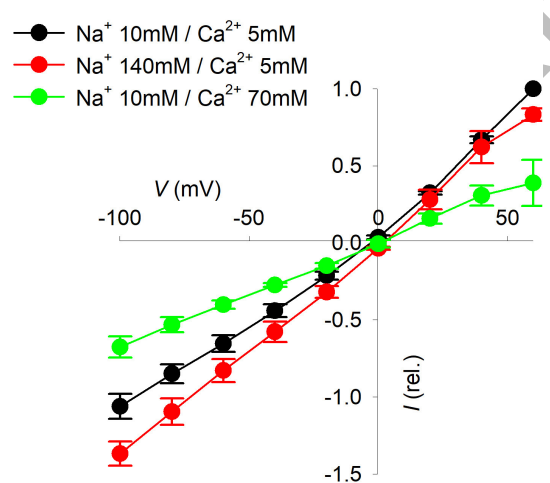

f

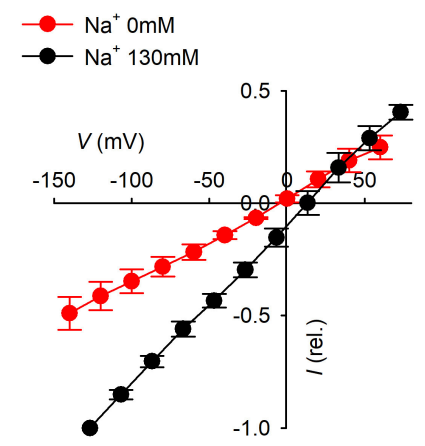

h
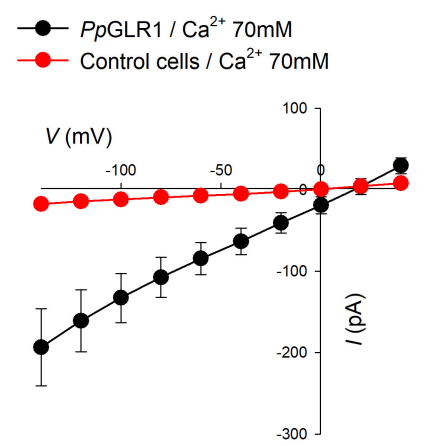

Extended Data Figure 6 | See next page for caption. 
Extended Data Figure 6 PpGLR1 is a non-selective channel. (a) Typical currents recorded in wild-type protoplasts from protonema with $\mathrm{Na}^{+}$ 10 or $110 \mathrm{mM}$. (b) Standardized current/ voltage curves of experiments in a. $\mathrm{Na}^{+} 10 \mathrm{mM}$ (black circles), $110 \mathrm{mM}$ (red circles) $(n=4)$. (c) Typical currents recorded in COS-7 cells transfected with pCI-PpGLR1 with external solutions containing (i) $\mathrm{Na}^{+} 10 \mathrm{mM}, \mathrm{Ca}^{2+} 5 \mathrm{mM}, \mathrm{Cl}^{-} 150 \mathrm{mM}$ (ii) $\mathrm{Na}^{+} 140 \mathrm{mM}, \mathrm{Ca}^{2+} 5 \mathrm{mM}, \mathrm{Cl}^{-} 150 \mathrm{mM}$ or (iii) $\mathrm{Na}^{+} 10 \mathrm{mM}, \mathrm{Ca}^{2+} 70 \mathrm{mM}$, $\mathrm{Cl}^{-} 150 \mathrm{mM}$. (d) Standardized current/ voltage curves of experiments presented in c. $\mathrm{Na}^{+} 10 \mathrm{mM}, \mathrm{Ca}^{2+} 5 \mathrm{mM}$ (black circles), $\mathrm{Na}^{+} 140 \mathrm{mM}, \mathrm{Ca}^{2+}$ $5 \mathrm{mM}$ (red circles), $\mathrm{Na}^{+} 10 \mathrm{mM}, \mathrm{Ca}^{2+} 70 \mathrm{mM}$ (green circles) $(n=3)$. (e) Representative current traces from COS-7 cells transfected with pCI-PpGLR1 in an external solution containing $\mathrm{Ca}^{2+} 10 \mathrm{mM}$ and $\mathrm{Cl}^{-}$ $10 \mathrm{mM}$ without $\mathrm{Na}^{+}$(left) and with Na-Gluconate $130 \mathrm{mM}$ (right) applied by perfusion. Here, pipette solutions were the same as Fig.4g, $h$.

(f) Standardized current/voltage curves of experiments shown in e. $\mathrm{Na}^{+}$free solutions (red circles), $\mathrm{Na}^{+} 130 \mathrm{mM}$ (black circles) $(\mathrm{n}=3)$. When increasing the external $\mathrm{Na}^{+}$concentration from 10 to $140 \mathrm{mM}$ a current increase was observed, demonstrating the permeability of PpGLR1 for
$\mathrm{Na}^{+}$shown in $\mathbf{c}$, d. Furthermore, $\mathrm{Na}^{+}$permeability was confirmed as the current intensity increased and the reversal potential also significantly shifted to the right when adding $\mathrm{Na}^{+}$to the external solution, as shown in e, f. (g) Representative current traces from control cell (left) and COS-7 cells transfected with pCI-PpGLR1 (right) in $\mathrm{Ca}^{2+} 70 \mathrm{mM}$ bathing solution (experimental conditions same as in Fig. 4g, h). (h) Current/voltage curve depicting average stationary current intensity as shown in $\mathbf{g}$, control (red circles) and pCI-PpGLR1(black circles) $(\mathrm{n}=3)$. The increase of current by external $\mathrm{Ca}^{2+}$ demonstrates a permeability of $P p$ GLR1 to $\mathrm{Ca}^{2+}$. Together with data presented in $\mathbf{c}$ and $\mathbf{d}$, these results suggest a complex effect of external $\mathrm{Ca}^{2+}$. Indeed, while $\mathbf{g}$ and $\mathbf{h}$ demonstrate $\mathrm{Ca}^{2+}$ permeation in conditions where no $\mathrm{Na}^{+}$and low $(6 \mathrm{mM}) \mathrm{Cl}^{-}$are present, $\mathbf{c}$ and $\mathbf{d}$ show a decrease of ionic currents by high extracellular $\mathrm{Ca}^{2+}$ when the bath contains $\mathrm{Na}^{+} 10 \mathrm{mM}$ and $\mathrm{Cl}^{-} 150 \mathrm{mM}$. Panels E- $\mathrm{H}$ also suggest that $\mathrm{Cl}^{-}$may pass through the channel accounting for the portion of outward current. All error bars represent standard error. In all cases $n$ represents biological replicates. 


\section{RESEARCH LETTER}

a

Ppglr1 scheme

PpGLR1 (Pp1s56_31v6.1)

b

Ppglr2 scheme

PpGLR2(Pp1s253_26v6.1)

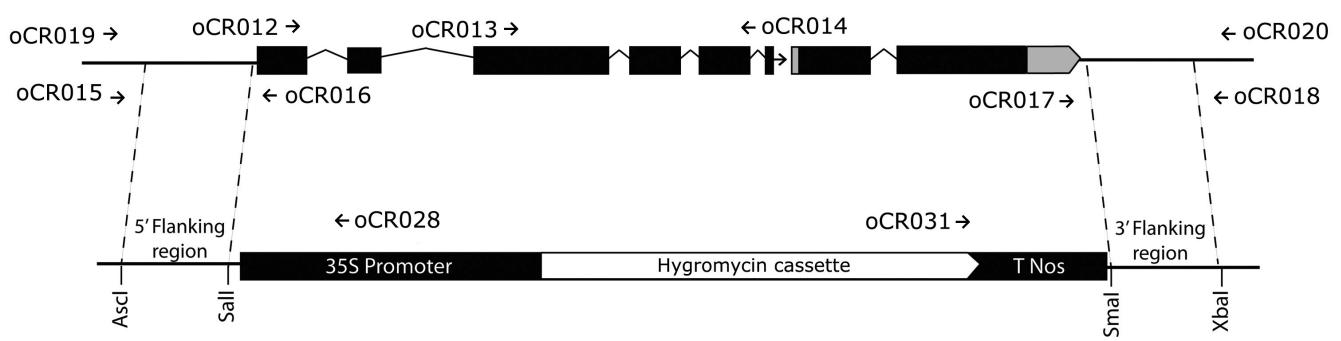

C
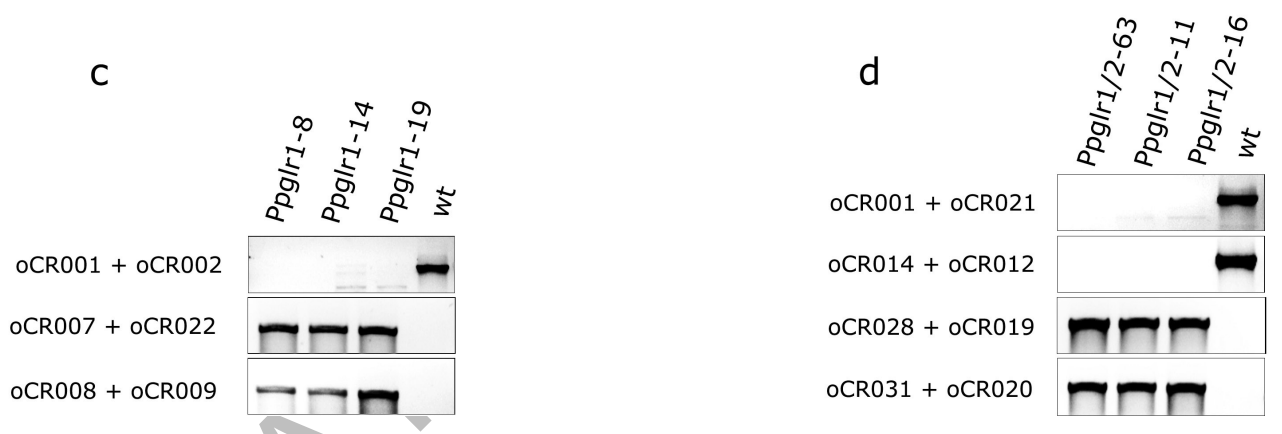

Extended Data Figure 7 | Schematic representation of the constructs used for the generation of Ppglr1 and Ppglr1/2 mutants by homologous recombination. (a) $P p G L R 1$ endogenous genomic locus (top line) and pBNRF plasmid used for transformation (bottom lines) are shown.

The boxes and lines between the boxes represent exons and introns respectively. The boxes coloured in grey represent untranslated regions (UTRs) of the gene and the small arrows indicate the approximate position were primers bind to the DNA sequence. Broken lines denote the gene's

flanking genomic regions used for homologous recombination. (b) The endogenous genomic locus of PpGLR2 (top line) and pBHrev construct used for transformation (bottom line) are also shown. (c) PCR showing the deletion of PpGLR1 gene from Ppglr1 knock out mutants and the correct insertion of the construct used for transformation. (d) PCR showing the same for Ppglr1/2 double knock out mutants (uncropped gel images are available as supplementary information). 
a

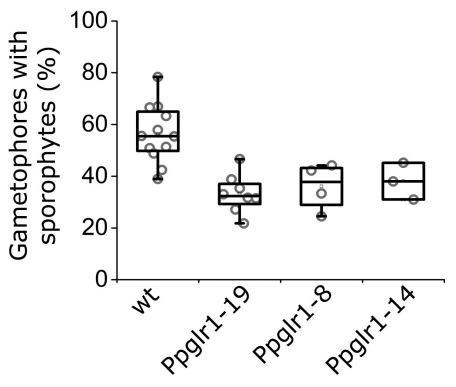

C

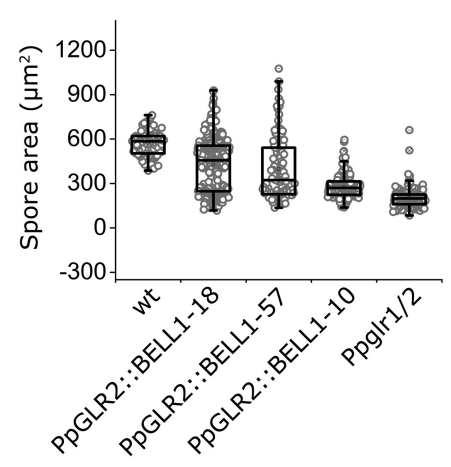

Extended Data Figure 8 | Independent mutant lines have similar phenotypes. (a) All Ppglr1 mutant lines show a significant difference in sporophyte production when compared to $w t$ ( $\mathrm{p} \leq 0.012$, two-tailed $t$-test). 100 gametophores were counted per sample (biological replicates), $n=12$ (wt), $n=8$ (Ppglr1-19), $n=4$ (Ppglr1-8), $n=3$ (Ppglr1-14). (b) Similarly, all Ppglr2 mutant lines produced very few sporophytes when compared to $w t$ ( $\mathrm{p} \leq 0.004$, Mann-Whitney $U$ test). Data from several biological replicates, $n=12(w t), n=9$ (Ppglr1/2-63), $n=7$ (Ppglr1/2-11), $n=4$ (Ppglr1/2-16). 100 gametophores were counted per sample. (c) All complementation lines had significantly bigger spores when compared to Ppglr1/2 double knock b

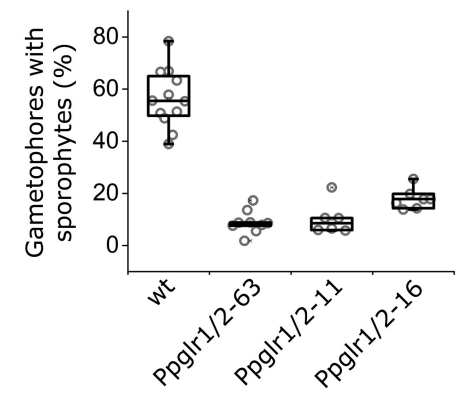

d

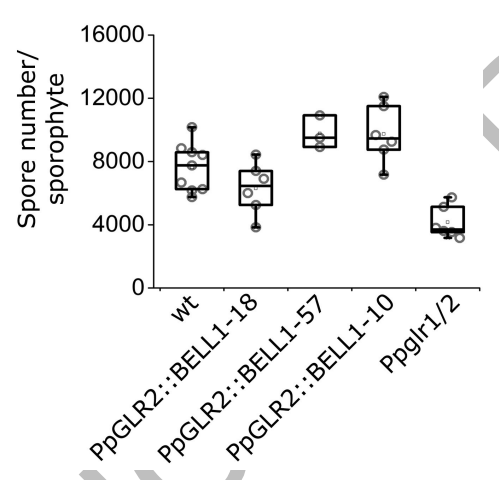

out ( $\mathrm{p} \leq 0.001$, Mann-Whitney $U$ test). Spores from 3 biological replicates were pulled and measured. Data points represent individually measured spores. $n=82(w t), n=174($ Ppglr1/2) $n=117$ (PpGLR2::BELL1-57), $n=284$ (PpGLR2::BELL1-18), $n=202$ (PpGLR2::BELL1-10). (d) Similarly, $P$ PGL2::BELL1 sporophytes clearly produced more spores than Ppglr1/2 ( $\mathrm{p} \leq 0.022$, two-tailed $t$-test). Data from several biological replicates. $\mathrm{n}=9$ (wt), $\mathrm{n}=6$ (PpGLR2::BELL1-18, PpGLR2::BELL1-10, Ppglr1/2), $\mathrm{n}=3$ (PpGLR2::BELL1-57). In all cases box plots show 25, 75 percentile and lines indicate outliers. 


\section{RESEARCH LETTER}

a

Ppglr2 scheme

PpGLR2 (Pp1s253_26v6.1)

pTN83

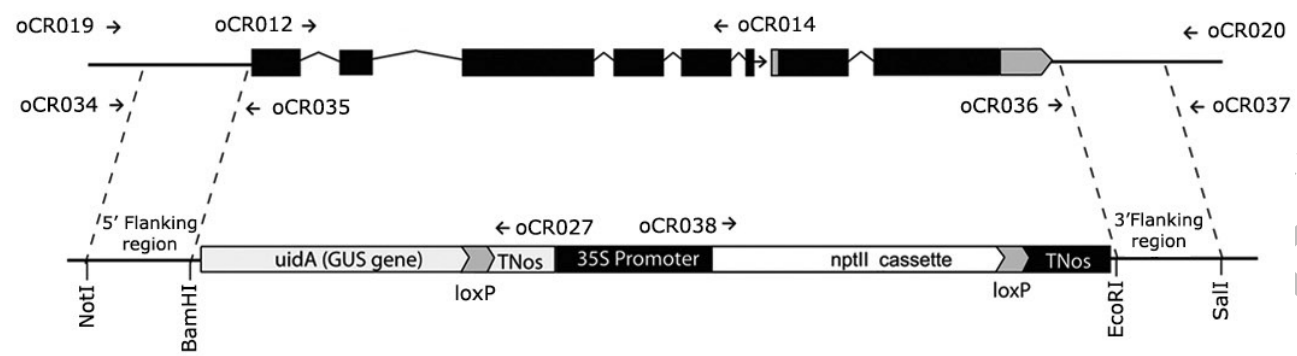

b

PpGLR2::PpBELL1 sheme

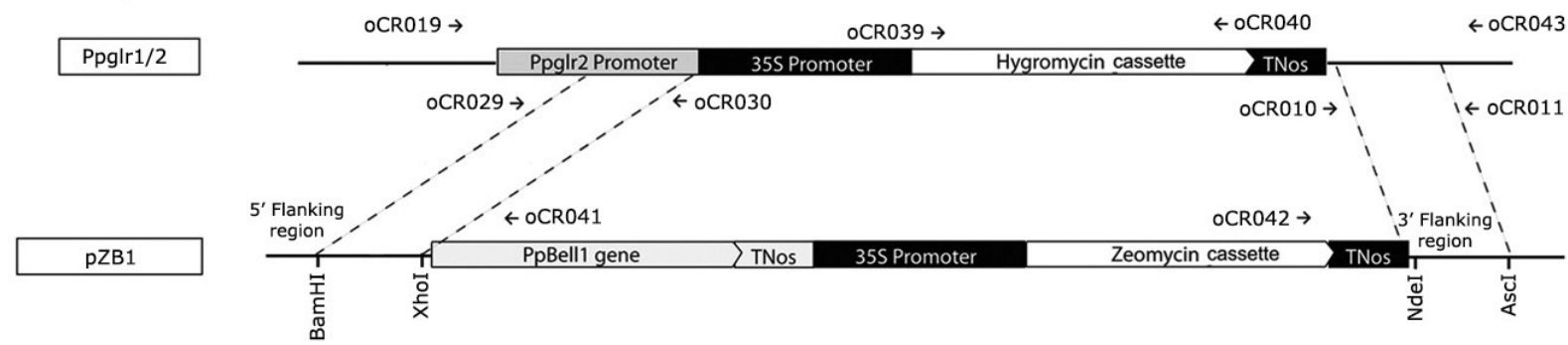

C

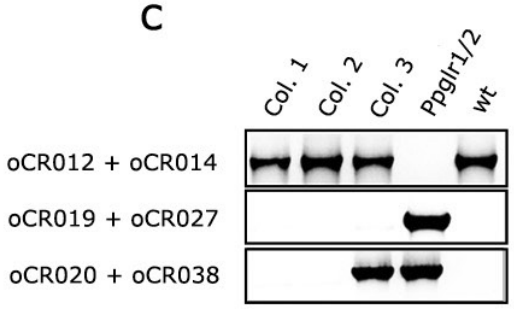

Extended Data Figure 9 | Schematic representation of the constructs used for the generation of Ppglr2 and PpGLR2::BELL1 mutants by homologous recombination. (a) $P p G L R 2$ endogenous genomic locus (top line) and pTN83 plasmid used for transformation (bottom lines) are shown. The boxes and lines between the boxes represent exons and introns respectively. Small arrows indicate the approximate position were primers bind to the DNA sequence. Broken lines denote the genomic regions used for homologous recombination. (b) The endogenous genomic locus in Ppglr1/2 mutant (top line) and pZB1 construct used for transformation (bottom line) are also shown. (c) PCR showing the deletion of $P p G L R 2$ d

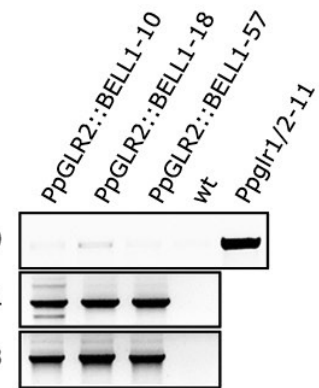

gene from Ppglr2 single knock out mutants and the correct insertion of the construct used for transformation. (d) Hygromycin could not be detected in PpGLR2::BELL1 complementation mutants and PCRs show correct insertion of the construct. (e) RT-PCRs experiments show that $P p G L R 1$ transcripts are more abundant in $35 s:: P p G L R 1$ lines. 25 cycles of PCR amplification using cDNA obtained from protonema were used to amplify PpGLR1 in $w t$ and overexpression lines. Tubulin was used as a control $(P p T U B)$ (uncropped gel images are available as supplementary information).

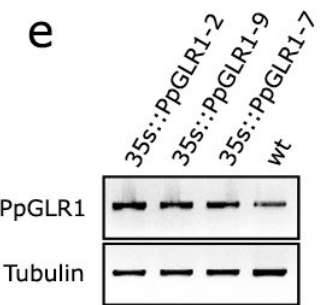


Extended Data Table 1 | Differentially expressed genes in Ppglr1/2

\begin{tabular}{llll}
\hline Gene ID & Fold change & At homolog & Description \\
\hline Pp1s56_31V6 & -67.37 & GLR3.3 & Glutamate receptor \\
Pp1s253_26V6 & -38.14 & GLR3.4 & Glutamate receptor \\
Pp1s455_4V6 & -8.35 & n/a & polyphenol oxidase \\
Pp1s258_6V6 & -7.69 & BLH7 & BELL1-like homeodomain \\
Pp1s79_259V6 & -5.03 & No homology & Oryza sativa GIL1, putative \\
Pp1s126_153 & -6.06 & No homology & \\
Pp1s18_45V6 & -4.18 & No homology & \\
Pp1s142_76V6 & -3.96 & No homology & \\
Pp1s385_31V6 & -3.04 & AAH & allantoate amidohydrolase \\
Pp1s252_5V6 & -4.97 & AT1G22900.1 & Disease resistance-responsive
\end{tabular}

Microarray expression data from $w t$ and Ppg/r1/2 was used to generate a list of differentially expressed genes. The genes with the highest negative fold change (down-regulated in Ppg/r1/2 with respect to $w t$ ) are presented. 


\section{natureresearch}

Corresponding author(s): José A. Feijó

Initial submission $\square$ Revised version

Xinal submission

\section{Life Sciences Reporting Summary}

Nature Research wishes to improve the reproducibility of the work that we publish. This form is intended for publication with all accepted life science papers and provides structure for consistency and transparency in reporting. Every life science submission will use this form; some list items might not apply to an individual manuscript, but all fields must be completed for clarity.

For further information on the points included in this form, see Reporting Life Sciences Research. For further information on Nature Research policies, including our data availability policy, see Authors \& Referees and the Editorial Policy Checklist.

\section{- Experimental design}

\section{Sample size}

Describe how sample size was determined

\section{Data exclusions}

Describe any data exclusions.

\section{Replication}

Describe whether the experimental findings were reliably reproduced.

\section{Randomization}

Describe how samples/organisms/participants were allocated into experimental groups.
Minimal sample size estimation was calculated using the Soper D. Statistics Calculators version 4.0 (http://www.danielsoper.com/statcalc/default.aspx) to ensure a statistical power level of at least 0.7 , a Cohen's $d$ of 0.5 , and a probability of 0.05 for a two-tailed t-test. For small samples, sample size effect was estimated taking into account the mean and standard deviation of samples used for these experiments using the Ellis PD. Effect size calculators (http://www.polyu.edu.hk/ $\mathrm{mm} /$ effectsizefaqs/calculator/calculator.html).

There was no data exclusion from our statistical analyses. All samples were considered including those that deviated from the sample mean "outliers".

For determining the sporophyte production rates (percentage of gametophore with sporophytes) one attempt out of 12 , failed to replicate the expected production rate in wt and PpgIr1 genotypes. However, after close examination we determined that the mentioned batch of samples had fungi contamination and was not used for data collection. The rest of the experiments presented in the paper were successfully replicated.

\section{Blinding}

Describe whether the investigators were blinded to group allocation during data collection and/or analysis.
No randomization method was used. Samples were not exposed to any treatments or stress conditions that deviate from what is considered the optimal growing conditions for Physcomitrella patens. Therefore randomized allocation of samples was not considered necessary. We rather focused on sample comparability, being careful to expose all the samples (genotypes) and experimental groups (single batch of samples or genotypes) to precisely the same growing and gametangia inducing conditions (i.e. temperature, light, humidity, nutrients, substract, growing material, examination conditions) for exactly the same amount of time. In addition, tissue dissection and data collection was performed after the same period of time for each of the replicates.

Since we did not impose treatments or stress conditions in our sample groups, we didn't consider blinding to be necessary. Moreover, to ensure that samples from different genotypes were exposed to the same conditions we preferred to have them labeled with the genetic background information. 


\section{Statistical parameters}

For all figures and tables that use statistical methods, confirm that the following items are present in relevant figure legends (or in the Methods section if additional space is needed).

n/a Confirmed

\ The exact sample size ( $n$ ) for each experimental group/condition, given as a discrete number and unit of measurement (animals, litters, cultures, etc.)

$\searrow^{A}$ description of how samples were collected, noting whether measurements were taken from distinct samples or whether the same

sample was measured repeatedly

$\searrow$ A statement indicating how many times each experiment was replicated

The statistical test(s) used and whether they are one- or two-sided (note: only common tests should be described solely by name; more) complex techniques should be described in the Methods section)

$\bigotimes$ A description of any assumptions or corrections, such as an adjustment for multiple comparisons

$\bigotimes$ The test results (e.g. $P$ values) given as exact values whenever possible and with confidence intervals noted

$\bigotimes$ A clear description of statistics including central tendency (e.g. median, mean) and variation (e.g. standard deviation, interquarrtile range)

$\square$ Clearly defined error bars

See the web collection on statistics for biologists for further resources and guidance.

\section{- Software}

Policy information about availability of computer code

\section{Software}

Describe the software used to analyze the data in this study.

dChip v2010.01 software was used for microarray analysis and determination of differentially expressed genes. Origin 9.0 and Sigma Plot v12.2 were used for statistical analyses. Image I V2 was used for statistical analyses. No custom code was developed for this study.

For manuscripts utilizing custom algorithms or software that are central to the paper but not yet described in the published literature, software must be made available to editors and reviewers upon request. We strongly encourage code deposition in a community repository (e.g. GitHub). Nature Methods guidance for providing algorithms and software for publication provides further information on this topic.

\section{- Materials and reagents}

Policy information about availability of materials

\section{Materials availability}

Indicate whether there are restrictions on availability of unique materials or if these materials are only available for distribution by a for-profit company.

\section{Antibodies}

Describe the antibodies used and how they were validated NA for use in the system under study (i.e. assay and species).

10. Eukaryotic cell lines

a. State the source of each eukaryotic cell line used.

b. Describe the method of cell line authentication used.

c. Report whether the cell lines were tested for mycoplasma contamination.

d. If any of the cell lines used are listed in the database of commonly misidentified cell lines maintained by ICLAC, provide a scientific rationale for their use.
There are no availability restrictions for material used in this study.

NA

NA

NA

NA 
Policy information about studies involving animals; when reporting animal research, follow the ARRIVE guidelines

11. Description of research animals

Provide details on animals and/or animal-derived materials used in the study.

NA

Policy information about studies involving human research participants

12. Description of human research participants

Describe the covariate-relevant population

NA

characteristics of the human research participants. 